\title{
Upgradient and Downgradient Potential Vorticity Fluxes Produced by Forced Rossby Waves. Part II: Parameter Sensitivity and Physical Interpretation
}

\author{
Genta MizUTA \\ Graduate School of Environmental Earth Science, Hokkaido University, Sapporo, Japan
}

(Manuscript received 24 September 2017, in final form 21 March 2018)

\begin{abstract}
We examine the potential vorticity (PV) flux produced by forced Rossby waves in a two-layer quasigeostrophic model, using a perturbation analysis. Rossby waves are excited by external forcing applied to the upper layer. The southward PV flux is produced in the lower layer by the higher-order Rossby waves that are excited by nonlinear wave-wave interactions, whereas the northward PV flux is produced in the upper layer. The direction of the PV flux is consistent with that obtained by an eddy-resolving model of the wind-driven circulation in previous studies. The southward PV flux is produced in a wide parameter range comparable to the eddy-resolving model. The basic features of the PV flux remain unchanged even in the limit of weak stratification. In this limit, stratification has nearly no effect on the flow, except that it isolates the lower layer from the direct effects of external forcing. The mechanism of the southward PV flux is explained using basic features of the barotropic Rossby waves and does not depend on details of the model. Furthermore, the resonant triad interaction of Rossby waves does not affect the PV flux. Stratification weakens or strengthens the PV flux depending on the horizontal scale of the external forcing.
\end{abstract}

\section{Introduction}

A western boundary current and its eastward extension, such as the Gulf Stream, Kuroshio, and Kuroshio Extension, are flanked by recirculation gyres (Kawai 1972; Worthington 1976; Hogg 1983; Hogg et al. 1986; Jayne et al. 2009). The volume transport of each of the recirculation gyres flanking the northern and southern sides of the Gulf Stream is $20-30 \mathrm{~Sv}\left(1 \mathrm{~Sv} \equiv 10^{6} \mathrm{~m}^{3} \mathrm{~s}^{-1}\right.$; Hogg 1992). The transport of the Gulf Stream is locally increased from about 95 to $150 \mathrm{~Sv}$ by the recirculation gyres (Hogg 1992). Pioneering numerical experiments by Holland and Rhines (1980) showed that the eddy potential vorticity (PV) flux produced by mesoscale perturbations drives recirculation gyres. Two possible mechanisms maintaining recirculation gyres have been proposed. One is PV homogenization (Rhines and Young 1982, hereinafter RY82). RY82 proposed that stirring of the PV by mesoscale perturbations drives the deep recirculation gyres. However, they assumed without proof that the PV flux is oriented in the downgradient direction of the time-mean PV.

The other possible mechanism is the rectification of Rossby wave motion, which is proposed by observational,

Corresponding author: Genta Mizuta, mizuta@ees.hokudai.ac.jp numerical, and theoretical studies (Thompson 1977; Hogg 1988; Malanotte-Rizzoli et al. 1995; Mizuta 2009; Waterman and Jayne 2011). Planetary and topographic Rossby waves tend to dominate in the mesoscale perturbations near the western boundary current at periods from a few tens of days to a few months (Thompson and Luyten 1976; Thompson 1977; Hogg 1981; Imawaki 1985; Bower and Hogg 1992). Haidvogel and Rhines (1983, hereinafter HR83) represented the strong perturbation of the western boundary current by an external forcing that is oscillatory in time and localized in space. They showed that Rossby waves excited by forcing drives a mean flow that is qualitatively the same as recirculation gyres. The advantage of the Rossby wave rectification mechanism is that the PV flux is determined by linear dynamics. This contrasts with the study by RY82, which assumed the downgradient PV flux without proof.

However, neither RY82 nor HR83 can completely reproduce the PV flux obtained in the numerical experiment by Holland and Rhines (1980). That is, the PV flux in Holland and Rhines (1980) is northward in the surface layer and southward in the deep layer, implying that the PV flux in the surface layer is oriented in the upgradient direction of the mean PV. Hence, the assumption by RY82 does not hold in the surface layer. The PV flux obtained by HR83, who used a barotropic 
model, is northward and only partly consistent with that of Holland and Rhines (1980). Thus, the mechanism maintaining the recirculation gyres has not been sufficiently understood. To resolve this difficulty, it is important to examine more precisely the character of the PV flux by Rossby waves. Waterman and Jayne (2012) extended the study by HR 83 and examined effects of the mean flow and the horizontal divergence on Rossby waves. However, the character of the PV flux by Rossby waves has not been fully understood.

In Mizuta (2018, hereinafter Part I), the southward PV flux was produced in the deep layer when stratification and nonlinearity were included in the HR 83 experiment, implying that the direction of the PV flux was consistent with that in Holland and Rhines (1980) in both the surface and deep layers. The southward PV flux is produced in both the linear case, in which linear theory of the wave-wave and wave-mean flow interaction is applicable to a good approximation, and in the nonlinear case, in which the nonlinear effect is of the same order as the beta effect. However, the mechanism producing the southward PV flux is still not clear enough. In addition parameters such as the horizontal scale and frequency of the forcing were fixed in Part I. The southward PV flux is produced by the harmonic wave, which is excited by nonlinearity of Rossby waves. Longuet-Higgins and Gill (1967) showed that Rossby waves can form a resonant triad, exchanging energy effectively between resonant waves through the nonlinear interaction. However, it is not clear if the resonant interaction affects the production of the PV flux. Hence, we further examine the meridional component of the PV flux produced by the Rossby wave in Part II.

In Part II, the PV flux produced by Rossby waves is examined for two purposes. The first purpose is to examine whether the southward PV flux in the deep layer is commonly produced in the wide parameter range. We focus on the perturbation analysis, which is shown in Part I to be able to qualitatively reproduce nonlinear effects even in a moderately nonlinear case. In Part I, we showed theoretically that the northward PV flux has to dominate when Rossby waves are excited by external or nonlinear forcing. Hence, it may be counterintuitive that the southward PV flux is produced by nonlinearity. The second purpose of Part II is to obtain a physical explanation of why the southward PV flux is produced. Part II is organized as follows: In section 2, we describe the formulation of the perturbation analysis. We describe the numerical method and results of the perturbation analysis in section 3 . We examine the mechanism producing the southward PV flux for a special case in which stratification is weak in section 4 and a more general case in section 5 . We summarize the results from these sections in section 6 .

\section{Formulation}

\section{a. Perturbation analysis}

We consider motions excited by external forcing that is oscillatory in time and localized in space in a two-layer, flat-bottomed ocean. It was shown in Part I that the basic feature of the PV flux is determined by the barotropic and the first baroclinic modes. Hence, we employed a twolayer model here for simplicity, instead of the Regional Ocean Model System (ROMS; Haidvogel et al. 2000) employed in Part I. By doing so, we can explore the distribution of PV flux in a wide range of parameters. We have confirmed that results of a two-layer model and ROMS are similar to each other if the same forcing and internal deformation radius are used.

When the quasigeostrophic approximation is assumed, the nondimensional equations of the PV are

$$
q_{k, t}+\varepsilon J\left(\psi_{k}, q_{k}\right)+2 \psi_{k, x}=2 W_{e}(x, y) e^{-i t} \delta_{k 1},
$$

where

$$
q_{k}=\nabla^{2} \psi_{k}+\frac{F}{2}(-1)^{k}\left(\psi_{1}-\psi_{2}\right)
$$

is the PV, $\psi_{k}$ is the streamfunction, $x$ and $y$ are the eastward and northward coordinates, respectively, $t$ is time, and the subscript $k=1,2$ denotes the upper- and lowerlayer variables, respectively. Because eddy motions, which are considered the source of the waves, are intense near the surface (Richardson 1983), the forcing on the right-hand side of (2.1) is confined to the upper layer. All variables have been scaled with a characteristic velocity $U$, the angular frequency $\omega$ of the forcing, and

$$
k_{\beta}=\frac{\beta}{2 \omega},
$$

which represents half of the maximum wavenumber of the barotropic Rossby wave at the frequency of $\omega$. The coefficient $\beta$ is the meridional gradient of the Coriolis parameter. The nondimensional parameter,

$$
\varepsilon=\frac{U k_{\beta}}{\omega},
$$

is a measure of nonlinearity, and

$$
F=\frac{1}{\left(r_{d}^{\operatorname{dim}} k_{\beta}\right)^{2}}
$$

corresponds to the reciprocal of the Burger number defined with $k_{\beta}$ and the internal deformation radius $r_{d}^{\mathrm{dim}}$ in dimensional units. For simplicity, we assume the upper- and lower-layer thicknesses to be the same. We 
discuss later that fundamental features of the PV flux do not change even if the ratio of the upper- and lowerlayer thicknesses takes a general value.

Assuming that $\varepsilon$ is small, we expand $\psi_{k}$ in an asymptotic series in $\varepsilon$ :

$$
\psi_{k}=\psi_{k}^{(0)}+\varepsilon \psi_{k}^{(1)}+\epsilon^{2} \psi_{k}^{(2)}+\cdots .
$$

Substituting (2.3) into (2.1) yields the $O(1)$ equations

$$
\begin{aligned}
\psi_{k}^{(0)} & =\psi_{k}^{p} e^{-i t} \quad \text { and } \\
-i q_{k}^{p}+2 \psi_{k, x}^{p} & =2 W_{e} \delta_{k 1},
\end{aligned}
$$

and the $O(\varepsilon)$ equations

$$
\begin{aligned}
\psi_{k}^{(1)} & =\psi_{k}^{h} e^{-2 i t}+\bar{\psi}_{k}^{(1)}, \\
-2 i q_{k}^{h}+2 \psi_{k, x}^{h} & =-\frac{1}{2} J\left(\psi_{k}^{p}, q_{k}^{p}\right) \equiv w_{k}^{h}, \quad \text { and } \\
2 \bar{\psi}_{k, x}^{(1)} & =-\frac{1}{2} J\left(\psi_{k}^{* p}, q_{k}^{p}\right) \equiv \bar{w}_{k}^{(1)},
\end{aligned}
$$

where

$$
q_{k}^{p}=\nabla^{2} \psi_{k}^{p}+\frac{F}{2}(-1)^{k}\left(\psi_{1}^{p}-\psi_{2}^{p}\right)
$$

The $q_{k}^{h}$ is determined from $\psi_{k}^{h}$ in the same manner as $q_{k}^{p}$, and the asterisk represents the complex conjugate. Equation (2.5) implies that the wave, which has the same frequency as the external forcing, is excited to the lowest order. This wave is referred to as the primary wave. Equations (2.7) and (2.8) imply that nonlinearity of the primary wave drives the mean flow $\bar{\psi}_{k}^{(1)}$ and the wave $\psi_{k}^{h}$, whose frequency is twice as large as that of the primary wave. We refer to $\psi_{k}^{h}$ as the harmonic wave. The harmonic wave drives the mean flow of $O\left(\varepsilon^{3}\right)$ :

$$
2 \bar{\psi}_{k, x}^{(3)}=-\frac{1}{2} J\left(\psi_{k}^{h^{*}}, q_{k}^{h}\right) \equiv \bar{w}_{k}^{(3)} .
$$

Because $O\left(\varepsilon^{2}\right)$ motions do not include the mean flow, the mean flow is dominated by $\bar{\psi}^{(3)}$ in the region where $\bar{\psi}^{(1)}$ is negligible. For the rest of Part II, we drop superscript $p$ for simplicity. Hence $\psi_{k}$ and $q_{k}$ without a superscript represent the streamfunction and $\mathrm{PV}$, respectively, of the primary wave.

By using the barotropic and baroclinic modes of $\psi$, which are respectively defined as

$$
\begin{aligned}
& \psi_{b}=\frac{\psi_{1}+\psi_{2}}{2} \text { and } \\
& \psi_{c}=\frac{\psi_{1}-\psi_{2}}{2},
\end{aligned}
$$

and $\psi_{b}^{h}$ and $\psi_{c}^{h}$, which are defined with $\psi_{k}^{h}$ in the same manner as $\psi_{b}$ and $\psi_{c}$, we can express (2.5) and (2.7) as

$$
\begin{aligned}
-i \nabla^{2} \psi_{b}+2 \psi_{b, x} & =W_{e}, \\
-i\left(\nabla^{2} \psi_{c}-F \psi_{c}\right)+2 \psi_{c, x} & =W_{e}, \\
-2 i \nabla^{2} \psi_{b}^{h}+2 \psi_{b, x}^{h} & =w_{b}^{h}, \quad \text { and } \\
-2 i\left(\nabla^{2} \psi_{c}^{h}-F \psi_{c}^{h}\right)+2 \psi_{c, x}^{h} & =w_{c}^{h} .
\end{aligned}
$$

The forcing to the harmonic wave is

$$
\begin{aligned}
& w_{b}^{h}=-J\left(\psi_{b}, q_{b}\right)-J\left(\psi_{c}, q_{c}\right) \quad \text { and } \\
& w_{c}^{h}=-J\left(\psi_{b}, q_{c}\right)-J\left(\psi_{c}, q_{b}\right),
\end{aligned}
$$

where

$$
\begin{aligned}
& q_{b}=\nabla^{2} \psi_{b} \quad \text { and } \\
& q_{c}=\nabla^{2} \psi_{c}-F \psi_{c}
\end{aligned}
$$

are the barotropic and baroclinic components of the PV, respectively. Equations (2.12) and (2.13) have homogeneous solutions, $\psi_{b}=A_{b} e^{i\left(k_{b} x+l_{b} y\right)}$ and $\psi_{c}=A_{c} e^{i\left(k_{c} x+l_{c} y\right)}$, which respectively satisfy the dispersion relation:

$$
\begin{aligned}
& \left(k_{b}+1\right)^{2}+l_{b}^{2}=1, \quad \text { and } \\
& \left(k_{c}+1\right)^{2}+l_{c}^{2}=1-F .
\end{aligned}
$$

Equation (2.21) indicates that wavenumbers $k_{c}$ and $l_{c}$ are complex; hence $\psi_{c}$ is evanescent when

$$
F>1 \text {. }
$$

Equation (2.22) is rewritten with variables in dimensional units as

$$
\omega>\omega_{c}=\frac{\beta r_{d}^{\mathrm{dim}}}{2} .
$$

The typical period of Rossby waves observed near the western boundary current is between a few tens of days and a few months (Thompson and Luyten 1976; Thompson 1977; Hogg 1981; Imawaki 1985; Bower and Hogg 1992), satisfying the condition in (2.23). Thus, we focus on the case $F>1$ in the present study. Note that both the primary and harmonic waves are evanescent when $F>1$.

\section{b. Meridional PV flux}

As we considered in Part I for the continuously stratified ocean, the direction of the PV flux is constrained by the enstrophy equation (Rhines and Holland 1979; 
Plumb 1986). Because this constraint is important in the present study, we briefly review it, focusing on the twolayer ocean. Equations (2.5) and (2.7) can be expressed in a general form,

$$
-i m q_{k}^{M}+2 \psi_{k, x}^{M}=w_{k}^{M},
$$

where $(m, M)=(1, p)$ or $(2, h)$ with $w_{k}^{p}=2 W_{e} \delta_{k 1}$. By multiplying (2.24) by $q_{k}^{*} / 4$, taking the real part, and introducing multiple time scales, we obtain the equation of enstrophy [or, more precisely, a wave action, because the denominator of the first term on the left-hand side of (2.25) corresponds to $2 \beta$ ]:

$$
\delta \frac{\partial}{\partial T} \frac{\left|q_{k}^{M}\right|^{2}}{4}+Y_{k}=\frac{1}{4} \operatorname{Re} w_{k}^{M} q_{k}^{M^{*}},
$$

where Re denotes the real part, $Y_{k}$ is the meridional component of the mean PV flux,

$$
\left(X_{k}, Y_{k}\right)=\operatorname{Re}\left(-\psi_{k, y}^{M} q_{k}^{M^{*}}, \psi_{k, x}^{M} q_{k}^{M^{*}}\right),
$$

and $\delta^{-1} T$ represents the time larger than $O(1)$. The second term on the left-hand side of (2.25) is rewritten as

$$
Y_{k}=\nabla \cdot \mathbf{J}_{H k}+(-1)^{k} J^{z}
$$

where

$\mathbf{J}_{H k}=\operatorname{Re}\left(\frac{\left|\psi_{k, x}^{M}\right|^{2}-\left|\psi_{k, y}^{M}\right|^{2}}{2}-\frac{F\left|\psi_{1}^{M}-\psi_{2}^{M}\right|^{2}}{4}, \psi_{k, x}^{M} \psi_{k, y}^{M *}\right)$ and

$$
J^{z}=\operatorname{Re} \frac{F}{4}\left(\psi_{1, x}^{M}+\psi_{2, x}^{M}\right)\left(\psi_{1}^{M^{*}}-\psi_{2}^{M^{*}}\right)
$$

are the horizontal and vertical components of the enstrophy flux, respectively. The right-hand side of (2.25) represents the production of enstrophy by $w_{k}^{M}$, whereas the first and second terms on the left-hand side of (2.25) represent the temporal change of enstrophy and the meridional PV flux, which is equivalent to the divergence of enstrophy flux. Equation (2.25) indicates that the northward and southward PV flux is produced when $w_{k}^{M}$ tends to increase and decrease enstrophy in the stationary state, respectively. No meridional PV flux is produced when $w_{k}^{M}$ vanishes corresponding to the conventional nonacceleration theorem.

Taking the summation over $k$ and integrating over a large area $S_{L},(2.27)$ yields

$$
\int_{S_{L}}\left(Y_{1}+Y_{2}\right) d x d y=\int_{\Gamma_{L}}\left(\mathbf{J}_{1 H}+\mathbf{J}_{2 H}\right) \cdot \mathbf{n} d l
$$

where $\Gamma_{L}$ is the boundary of $S_{L}$ and $\mathbf{n}$ is the unit vector normal to $\Gamma_{L}$, pointing outward. If motions are locally approximated by a plane wave on $\Gamma_{L}$, the enstrophy flux across $\Gamma_{L}$ is expressed in terms of the group velocity $\mathbf{c}_{g}$ :

$$
\mathbf{J}_{1 H}+\mathbf{J}_{2 H}=\mathbf{c}_{g} \frac{\left|q_{1}^{M}\right|^{2}+\left|q_{2}^{M}\right|^{2}}{4}
$$

(Plumb 1986). If there is no source of enstrophy outside of $S_{L}, \mathbf{c}_{g} \cdot \mathbf{n}$ is positive. Then (2.30) implies that the sum of the upper- and lower-layer PV fluxes integrated over $S_{L}$ is northward. Therefore, the northward PV flux has to dominate in $S_{L}$, whereas the PV flux can locally be southward in the region where $w_{k}$ tends to decrease the enstrophy.

For later use, we derive an alternative expression of the meridional PV flux. That is, eliminating $q_{k}^{M}$ from (2.24) and (2.25) yields

$$
Y_{k}=-\frac{1}{2} \operatorname{Re} w_{k}^{M^{*}} \eta_{k}^{M},
$$

where

$$
\eta_{k}^{M}=\frac{i \psi_{k, x}^{M}}{m}
$$

is the northward displacement of the fluid particles.

\section{Numerical experiment}

Equations (2.12)-(2.15) were solved numerically, using a Green's function:

$\psi_{b}(x, y)=\iint_{S} G_{b 1}\left(x-x^{\prime}, y-y^{\prime}\right) W_{e}\left(x^{\prime}, y^{\prime}\right) d x^{\prime} d y^{\prime}$

$\psi_{c}(x, y)=\iint_{S} G_{c 1}\left(x-x^{\prime}, y-y^{\prime}\right) W_{e}\left(x^{\prime}, y^{\prime}\right) d x^{\prime} d y^{\prime}$,

$\psi_{b}^{h}(x, y)=\iint_{S} G_{b 2}\left(x-x^{\prime}, y-y^{\prime}\right) w_{b}^{h}\left(x^{\prime}, y^{\prime}\right) d x^{\prime} d y^{\prime}, \quad$ and

$\psi_{c}^{h}(x, y)=\iint_{S} G_{c 2}\left(x-x^{\prime}, y-y^{\prime}\right) w_{c}^{h}\left(x^{\prime}, y^{\prime}\right) d x^{\prime} d y^{\prime}$

where $G_{b m}$ and $G_{c m}(m=1,2)$ are Green's functions defined as

$$
\begin{aligned}
G_{b m} & =\frac{1}{4 m} H_{0}^{(2)}\left(\frac{r^{\prime}}{m}\right) e^{i\left[\left(x-x^{\prime}\right) / m\right]}, \\
G_{c m} & =\frac{1}{4 m} H_{0}^{(2)}\left(i \kappa_{m} r^{\prime}\right) e^{i\left[\left(x-x^{\prime}\right) / m\right]}, \\
\kappa_{m} & =\sqrt{F-\frac{1}{m^{2}}}, \quad \text { and }
\end{aligned}
$$




$$
r^{\prime}=\sqrt{\left(x-x^{\prime}\right)^{2}+\left(y-y^{\prime}\right)^{2}} .
$$

The $H_{0}^{(2)}$ is the zeroth-order Hankel's function of the second kind and $S$ is the model domain, which is large enough compared to the horizontal scale of the forcing. Nonlinear forcings in (3.3) and (3.4) were obtained from (2.16) and (2.17). The external forcing $W_{e}$ in (3.1) and (3.2) was

$$
W_{e}=A e^{-[(|x|+|y|) / a]} .
$$

Because $W_{e}$ given by (3.5) has a single sign in space, the horizontal average of $W_{e}$ does not vanish. If $W_{e}$ changes its sign in space, the meridional PV flux also tends to change sign even in the linear barotropic model (Mizuta 2014). Because we are interested in the effects of nonlinearity and stratification on the direction of the PV flux, we chose the form of (3.5) to avoid complexity. In addition, $x$ and $y$ derivatives of $W_{e}$ are discontinuous along $x=0$ and $y=0$. However, we discuss later that this does not cause any problems.

Equations (3.1)-(3.4) include two independent parameters, $a$ and $F^{1 / 2}$, which represent the horizontal scale of the forcing scaled with $k_{\beta}^{-1}$ and the reciprocal of the internal deformation radius scaled with $k_{\beta}^{-1}$, respectively. We performed 51 experiments, changing $a$ from 0.25 to 3 for moderate $F^{1 / 2}$ values (first and second rows in Table 1) and from 0.5 to 1 for large $F^{1 / 2}$ values (the third row in Table 1 ). When $F^{1 / 2}$ is large, the internal deformation radius becomes small; hence experiments for large values of $a$ were not performed, as the number of grid boxes necessary to resolve the deformation radius becomes quite large in such experiments. The amplitude $A$ of the forcing does not affect the pattern of the primary and harmonic waves. Because the velocity scale $U$ was determined by the amplitude $A^{\mathrm{dim}}$ of the forcing in dimensional units as $U=$ $A^{\mathrm{dim}} / 12.5 k_{\beta} \omega$ in all experiments, we obtain $A=12.5$. Here the factor 12.5 is determined empirically so that $U$ represents the typical velocity. [It is shown from (3.1) and (3.3) that $\psi_{b}$ and $\psi_{b}^{h}$ do not depend significantly on $a$ for $a \gg 1$, as $G_{b m}$ attenuates with the horizontal scale of $O(1)$. Hence the appropriate scale of velocity $U$ can be obtained using a constant $A$ for $a \geq O(1)$. Although this is not the case for $a \ll 1, A=12.5$ was used in all experiments to avoid complexity.]

We performed numerical calculation with variables with dimensional units and nondimensionalized results for analysis. In all experiments, the internal deformation radius $r_{d}^{\mathrm{dim}}$ was $43 \mathrm{~km}$, which is a typical value near the Kuroshio Extension. We changed $F$ by changing $\omega$, which affects $k_{\beta}$ in (2.2). For a typical example, when the
TABLE 1. Experimental conditions. Experiments were performed for all possible pairs of $a$ and $F^{1 / 2}$ listed in the first and second rows, respectively. Two experiments with $a=0.5$ and 1 were performed for each $F^{1 / 2}$ listed in the third row.

$$
\begin{array}{ll}
a & 0.25,0.5,1,1.5,2,2.5,3 \\
F^{1 / 2} & 1.06,1.13,1.70,2.55,3.40 \\
& 4.24,5.09,5.94,6.79,7.64,8.49,9.34,10.19
\end{array}
$$

period of forcing is 100 days, $k_{\beta}^{-1}$ is $72.7 \mathrm{~km}$ and $F^{1 / 2}=$ 1.70. The horizontal scale $a k_{\beta}^{-1}$ of the forcing in dimensional units ranged between 11.4 and $436 \mathrm{~km}$. The dimension of the model domain was $4000 \mathrm{~km}$ by $4000 \mathrm{~km}$ for most of the experiments and $8000 \mathrm{~km}$ by $8000 \mathrm{~km}$ when $a k_{\beta}^{-1}$ was large. The number of grid boxes was 512 in both $x$ and $y$. We confirmed that the numerical solution does not substantially depend on the number of grids or the domain size.

As a typical example of the primary wave, Figs. 1a, $1 \mathrm{~b}$, and $1 \mathrm{~d}$ show the horizontal distribution of $\operatorname{Re} \psi_{1}$, $\operatorname{Re} \psi_{2}$, and $W_{e}$ for $a=1$ and $F^{1 / 2}=1.70$, respectively. The region away from the forcing is not shown because the PV flux is weak there. Because $\psi_{c}$ is evanescent, $\psi_{b}$ dominates in $\psi_{1}$ and $\psi_{2}$ except for the region near the forcing. The distribution of $\psi_{1}$ and $\psi_{2}$ is qualitatively the same as in HR83. Figure 1c shows the distribution of the northward PV flux by the primary wave in the upper layer. As we considered in section $2 b$, the PV flux is northward in the upper layer. No meridional PV flux is produced in the lower layer because the external forcing vanishes there (not shown). As a typical example of the harmonic wave, the horizontal distribution of $\operatorname{Re} \psi_{1}^{h}$ and $\operatorname{Re} \psi_{2}^{h}$ for the same experiment as in Fig. 1 is shown in Figs. $2 a$ and 2b, respectively. Although the amplitude of $\psi_{1}^{h}$ is large near $(x, y)=(0,0)$ owing to the contribution by $\psi_{c}^{h}$ (Fig. 2a), radiation of the barotropic wave is apparent in $\psi_{2}^{h}$ (Fig. 2b). Note that the contour intervals are different between Figs. 2a and $2 \mathrm{~b}$. Figures $2 \mathrm{c}$ and $2 \mathrm{~d}$ show the PV flux by the harmonic wave in the upper and lower layers, respectively. The PV flux is northward and southward in the upper and lower layers, respectively. Because the meridional PV flux by the primary wave vanishes in the lower layer, the southward PV flux by the harmonic wave dominates there.

The meridional distributions of zonally integrated PV fluxes in the lower layer for $a=0.25,0.5,1,2$, and 3 are compared in Figs. 3a-e for various values of $F^{1 / 2}$. The southward PV flux dominates in the lower layer for all values of $a$ and $F^{1 / 2}$. The weak northward PV flux is also produced near $y \sim 0$ for $F^{1 / 2}=1.13$, especially for small values of $a$ (purple lines in Figs. 3a,b). Although figures are not shown, $\psi_{c}$ increases as $F^{1 / 2} \rightarrow 1$, producing the 
(a)

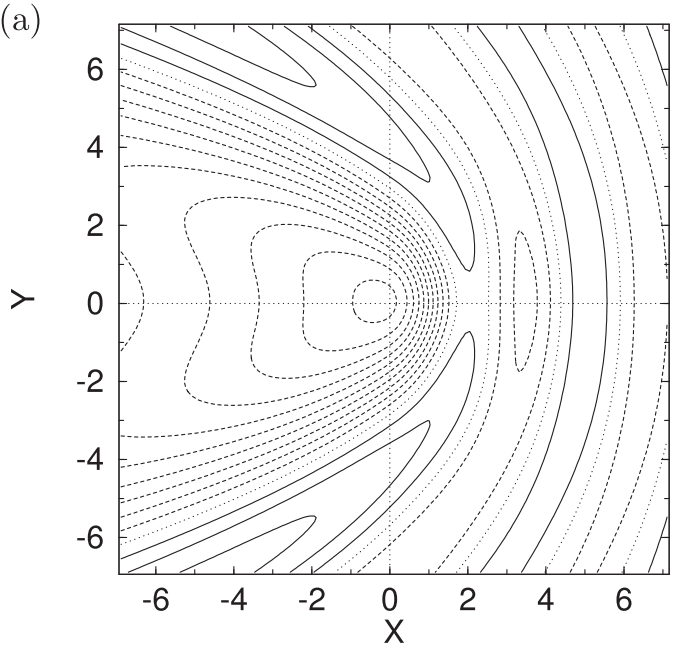

(c)

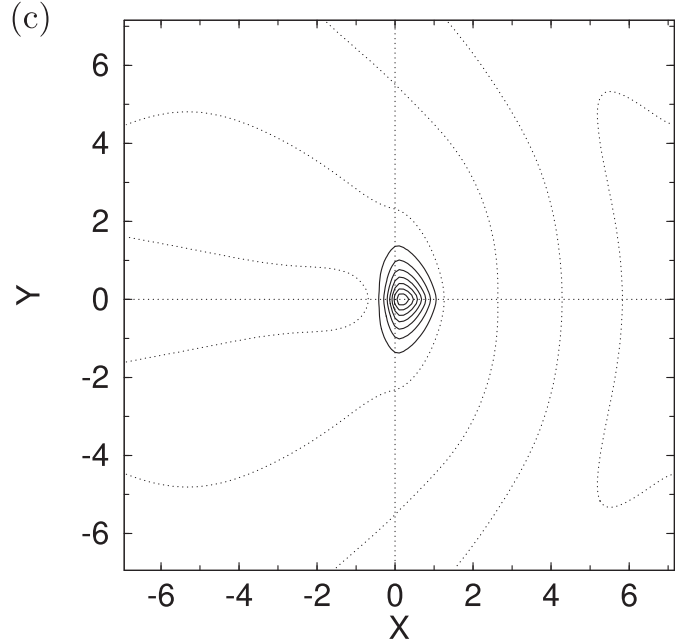

(b)

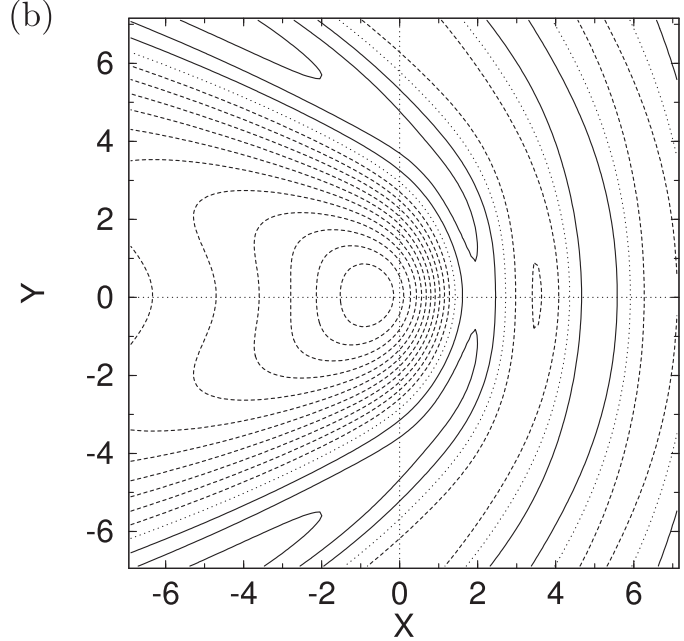

(d)

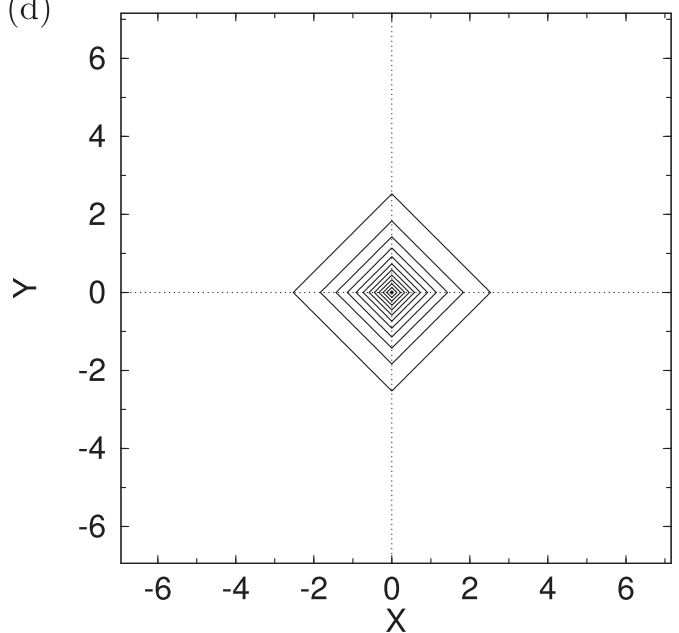

FIG. 1. The primary wave for $a=1$ and $F^{1 / 2}=1.70$. Contours of (a) $\operatorname{Re} \psi_{1}$, (b) $\operatorname{Re} \psi_{2}$, (c) the meridional PV flux in the upper layer, and (d) $W_{e}$. Solid and dashed contours indicate positive and negative values, respectively. Contour intervals are (a) 0.5 , (b) 0.5 , (c) 5 , and (d) 2 .

northward PV flux to the next order. However, $\psi_{c}$ increases only logarithmically as $F^{1 / 2} \rightarrow 1$.

As a measure of the amplitude of the southward PV flux in the lower layer, the minimum value of the zonally integrated PV flux $Y_{\min }$ is plotted against $F^{1 / 2}$ and $a$ in Figs. $4 \mathrm{a}$ and $4 \mathrm{~b}$, respectively. As $F^{1 / 2}$ increases, $Y_{\min }$ asymptotically approaches to a constant for both $a=0.5$ and 1 (Fig. 4a). Thus, the substantial amount of the southward PV flux is produced even if stratification is very weak. The horizontal lines in Fig. 4a indicate $Y_{\min }$ in the limit as $F^{1 / 2} \rightarrow \infty$, which will be derived in the next section. As $F^{1 / 2}$ increases, $Y_{\min }$ is asymptotic to these lines. The cyan, red, and black lines in Fig. 4b indicate $Y_{\min }$ for $F^{1 / 2}=1.70,3.40$, and $\infty$, respectively. The PV flux $Y_{\min }$ increases in amplitude with $a$ in $a<2.5$ and slightly decreases in $a>2.5$. In the limit $F \rightarrow \infty, Y_{\min }$ remains the same order as that for a finite value of $F^{1 / 2}$ even when $a$ takes various values.

As a measure of the horizontal scale of the PV flux, the latitude $y_{\min }$ of the minimum of the zonally integrated PV flux is plotted against $a$ in Fig. 5. Except for the case $F^{1 / 2}=1.13, y_{\min }$ is nearly proportional to $a$ (cyan, orange, and red lines in Fig. 5). The purple, cyan, orange, and red vertical lines in Fig. 5 indicate the lines $a=F^{-1 / 2}$ for $F^{1 / 2}=1.13,1,70,2.55$, and 3.40 , respectively. The change in $y_{\min }$ with $a$ is less significant, when $a<F^{-1 / 2}$ for $F^{1 / 2}=1.13$ (purple line in Fig. 5). The effects of $a$ and $F$ on the primary wave, which determines the PV flux in the upper layer to the lowest order, have already been examined by Waterman and Jayne (2012) and are not examined here. 
(a)

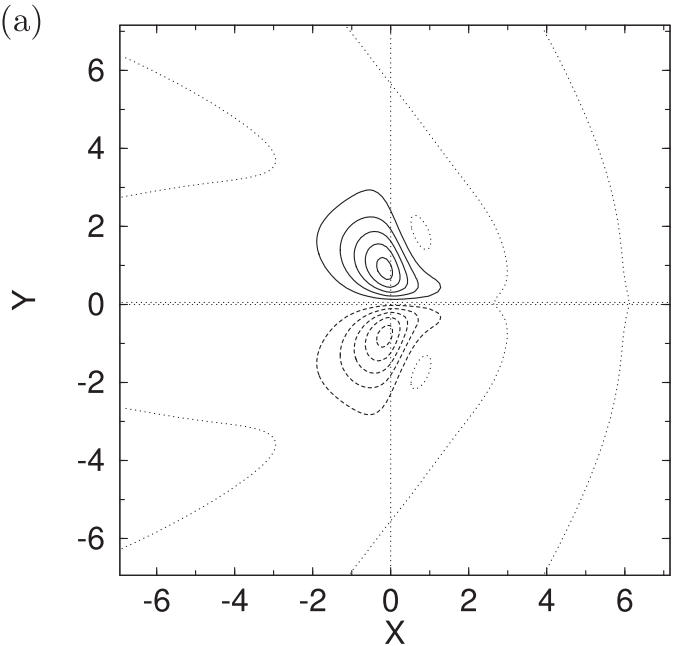

(c)

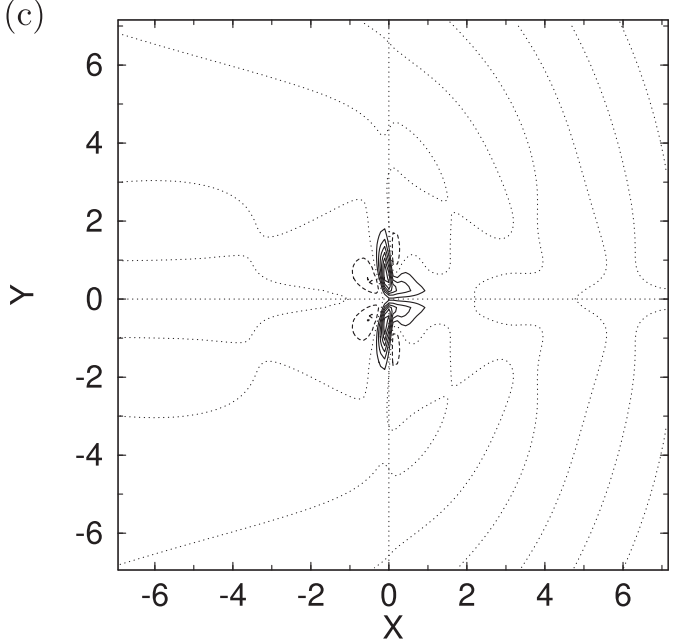

(b)

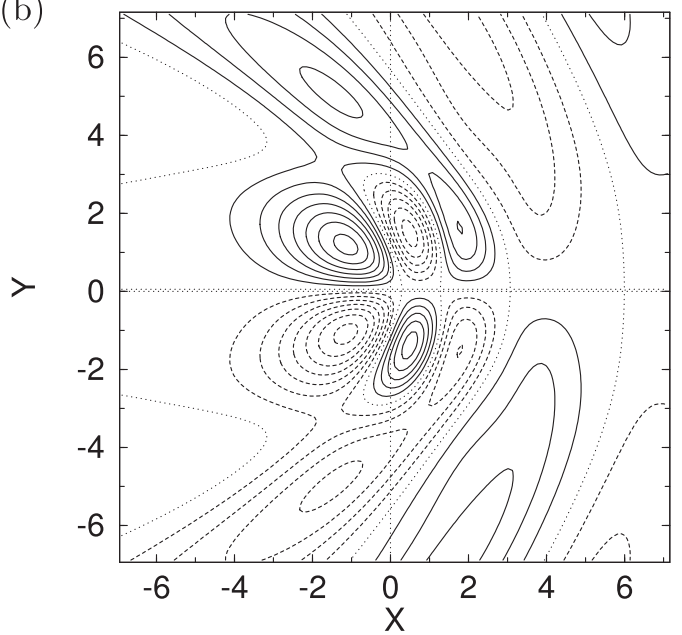

(d)

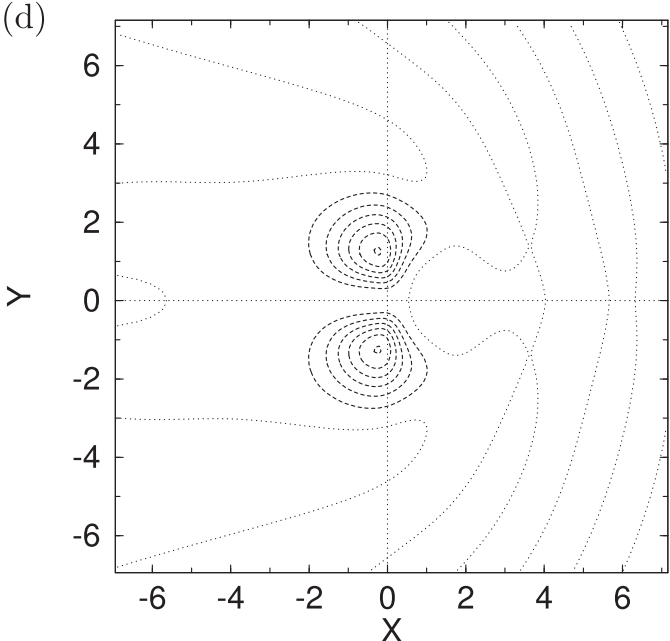

FIG. 2. The harmonic wave for $a=1$ and $F^{1 / 2}=1.70$. Contours of (a) $\operatorname{Re} \psi_{1}^{h}$, (b) $\operatorname{Re} \psi_{2}^{h}$, and the meridional PV flux in the (c) upper and (d) lower layers. Contour intervals are (a) 0.25 , (b) 0.05 , (c) 1, and (d) 0.05 .

\section{Weak stratification limit}

\section{a. Formulation}

Based on the results in the previous section, we consider the mechanism of the southward PV flux, focusing on the limit as $F \rightarrow \infty$. Expanding $\psi$ and $q$ in (2.12), (2.13), (2.18), and (2.19) in an asymptotic series of $F^{-1}$,

$$
\begin{aligned}
& \psi_{k}=\psi_{k}^{0}+F^{-1} \psi_{k}^{\prime}+F^{-2} \psi_{k}^{\prime \prime}+\cdots \quad \text { and } \\
& q_{k}=q_{k}^{0}+F^{-1} q_{k}^{\prime}+F^{-2} q_{k}^{\prime \prime}+\cdots,
\end{aligned}
$$

we obtain

$$
\begin{aligned}
& \psi_{c}^{0}=0, \\
& q_{c}^{0}=-\psi_{c}^{\prime}=i W_{e}, \\
& \psi_{b}^{\prime}=\psi_{b}^{\prime \prime} \cdots=0, \text { and }
\end{aligned}
$$

$$
q_{b}^{\prime}=q_{b}^{\prime \prime \cdots}=0 .
$$

Equations (4.3) and (4.4) indicate that the baroclinic mode contributes only to the change in $q_{k}$ without changing $\psi_{k}$ to the lowest order, and (4.5) and (4.6) indicate that $\psi_{b}=\psi_{b}^{0}$ and $q_{b}=q_{b}^{0}$.

Using (2.12), we can decompose $q_{b}$ into two components:

$$
\begin{aligned}
& q_{b}=q_{w}+q_{\beta}, \quad \text { and } \\
& q_{w}=i W_{e}, q_{\beta}=-2 i \psi_{b, x},
\end{aligned}
$$

where $q_{w}$ and $q_{\beta}$ represent the change in $q_{b}$ caused by external forcing and beta effect, respectively. Combining (4.4), (4.6), and (4.8), we obtain

$$
q_{1}^{0}=q_{b}+q_{c}^{0}=q_{b}+q_{w} \text { and }
$$


(a)

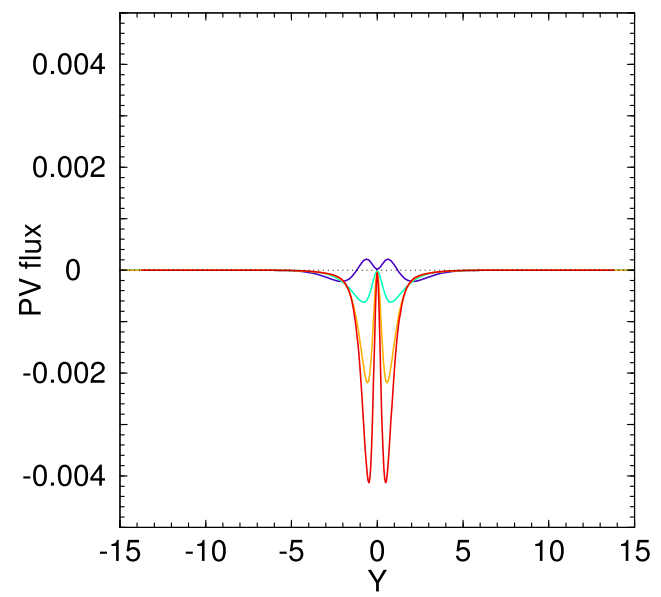

(c)

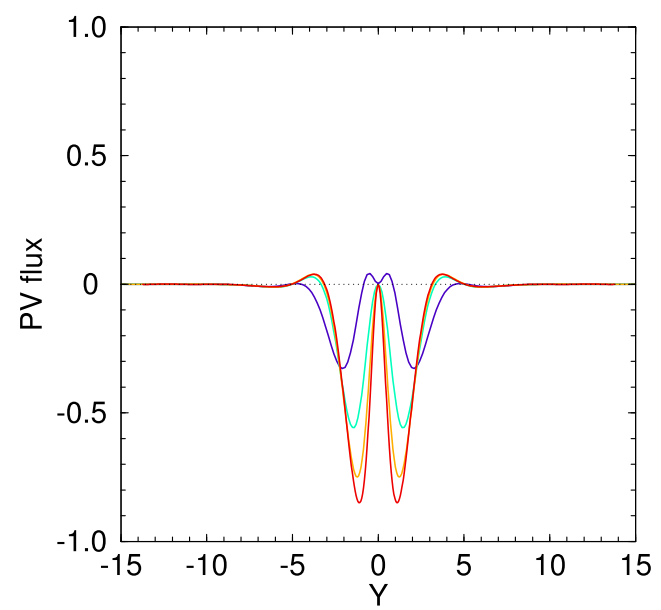

(b)

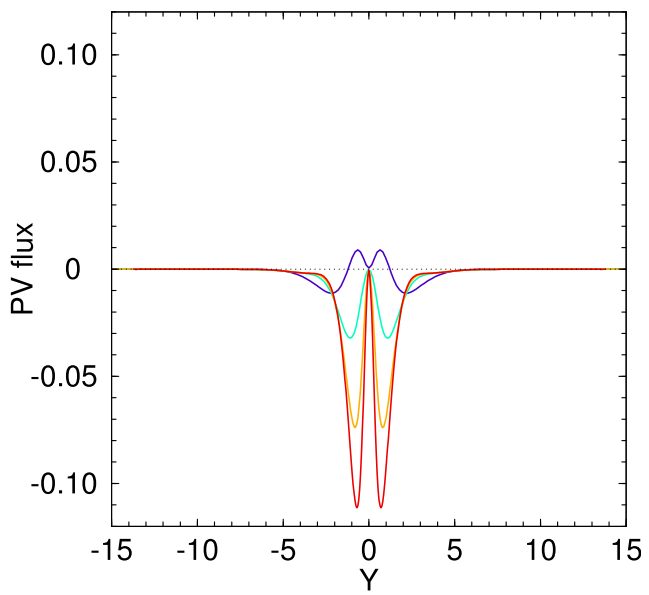

(d)

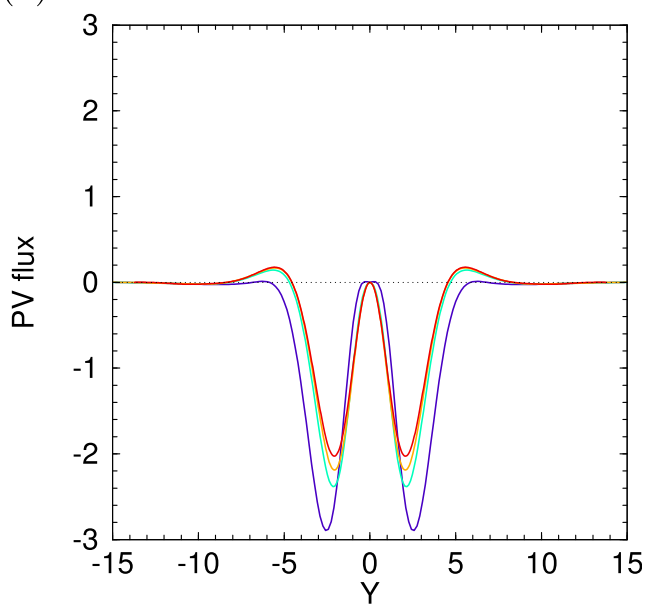

(e)

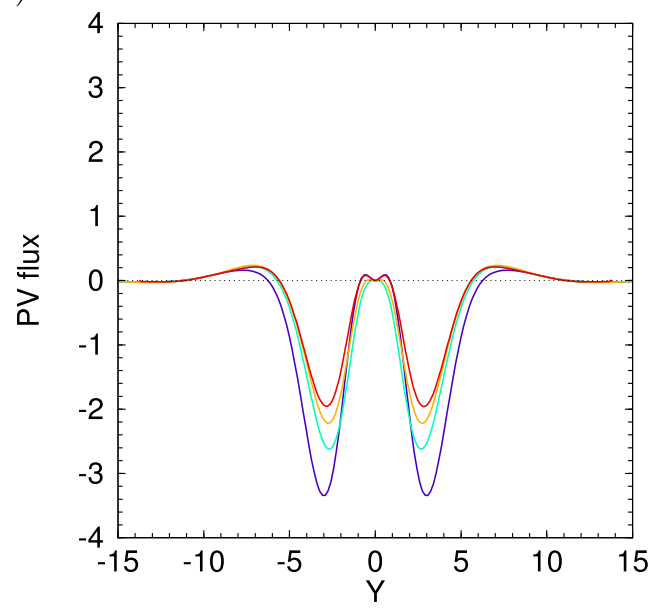

FIG. 3. Meridional distribution of the zonally integrated PV flux in the lower layer for $a=$ (a) 0.25 , (b) 0.5 , (c) 1 , (d) 2, and (e) 3. The purple, cyan, orange, and red lines indicate the PV flux for $F^{1 / 2}=1.13,1.70,2.55$, and 3.40, respectively. 
(a)

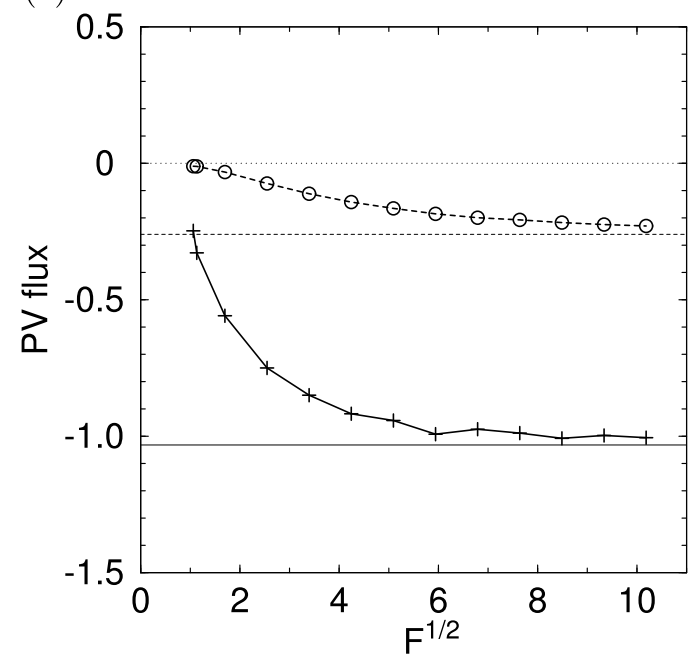

(b)

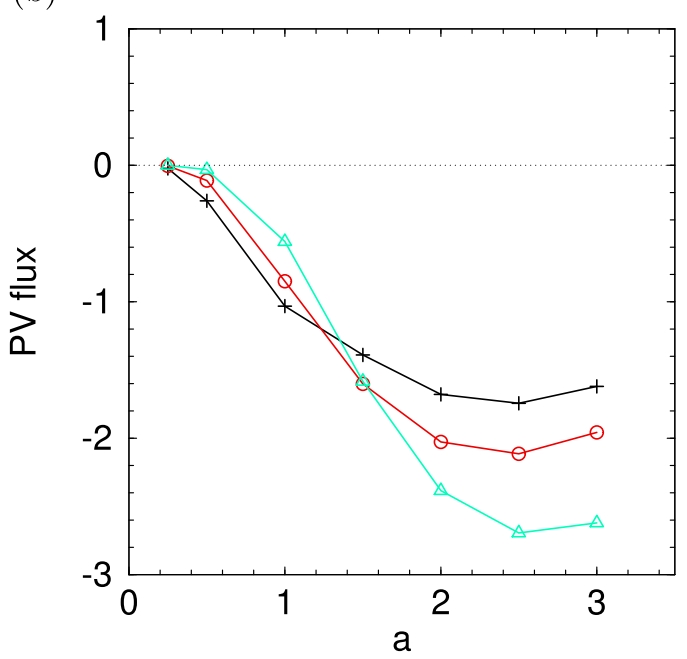

FIG. 4. (a) Plot of $Y_{\min }$ as a function of $F^{1 / 2}$ for $a=1$ (solid line with crosses) and 0.5 (dashed line with circles). The solid and dashed horizontal lines indicate $Y_{\min }$ in the limit as $F \rightarrow \infty$ for $a=1$ and 0.5, respectively. (b) Plot of $Y_{\min }$ as a function of $a$ for $F^{1 / 2}=1.70$ (cyan line with triangles), 3.40 (red line with circles), and $\infty$ (black line with crosses).

$$
q_{2}^{0}=q_{b}-q_{c}^{0}=q_{\beta} .
$$

Equation (4.10) indicates that the $\mathrm{PV} q_{2}^{0}$ in the lower layer is equivalent to $q_{b}$ except that it does not include the term $q_{w}$, which represents the direct contribution of external forcing.

Then, the forcing term on the right-hand side of (2.7) is expressed as

$$
\begin{aligned}
& w_{1}^{h 0}=2 W-W_{\beta} \quad \text { and } \\
& w_{2}^{h 0}=W_{\beta},
\end{aligned}
$$

where

$$
W=-J\left(\psi_{b}, q_{b}\right), \quad W_{\beta}=-J\left(\psi_{b}, q_{\beta}\right),
$$

represent the nonlinear forcing due to the advection of $q_{b}$ and $q_{\beta}$ by $\psi_{b}$, respectively, and the superscript 0 denotes the $O(1)$ variables.

As in the primary wave, $\psi_{c}^{h}$ is $O\left(F^{-1}\right)$. Then, using (2.31), the PV flux in the lower layer and the barotropic component of the PV flux by the harmonic wave, respectively, are

$$
\begin{aligned}
& Y_{2}^{h 0}=-\frac{1}{2} \operatorname{Re} \eta_{b}^{h 0} W_{\beta}^{*} \quad \text { and } \\
& Y_{b}^{h 0}=\frac{1}{2}\left(Y_{1}^{h 0}+Y_{2}^{h 0}\right)=-\frac{1}{2} \operatorname{Re} \eta_{b}^{h 0} W^{*},
\end{aligned}
$$

where

$$
\eta_{b}^{h 0}=\frac{i \psi_{b, x}^{h 0}}{2}
$$

is the barotropic component of the northward displacement of fluid particles by the harmonic wave. As considered in section $2 \mathrm{~b}, Y_{b}^{h 0}$ has to be northward in the sense that it is integrated over the entire domain. Thus, comparing (4.14) and (4.15) indicates that $Y_{2}^{h 0}$ becomes southward when $W_{\beta}$ is out of phase with $W$. It can be readily shown that the above argument remains qualitatively the same, even if the

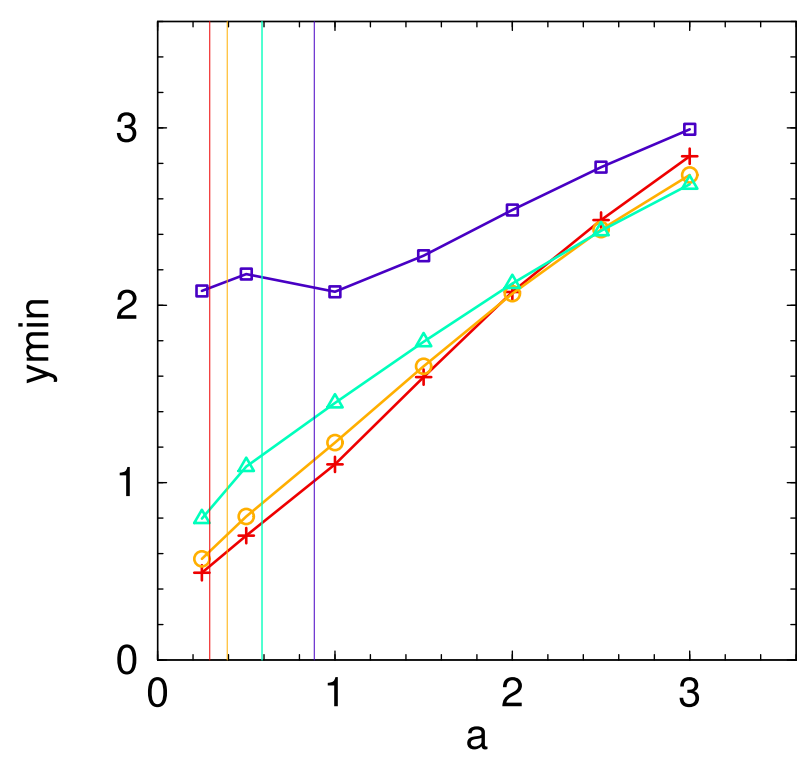

FIG. 5. Plot of $y_{\min }$ as a function of $a$ for $F^{1 / 2}=1.13$ (purple line with squares), 1.70 (cyan line with triangles), 2.55 (orange line with circles), and 3.40 (red line with crosses). The vertical purple, cyan, orange, and red lines indicate the lines $a=F^{1 / 2}$ for $F^{1 / 2}=1.13,1.70$, 2.55 , and 3.40 , respectively. 
ratio of the upper- and lower-layer thicknesses takes a general value.

Although we have considered a nearly barotropic ocean with weak stratification in this section, the PV is not barotropic. This might not seem physically consistent. Hence, before proceeding, we check the equation of enstrophy considered in section $2 b$ in the limit as $F \rightarrow \infty$. For the primary wave, (2.25) is rewritten as

$$
\begin{aligned}
\nabla \cdot \mathbf{J}_{H k} & =(-1)^{k-1} J^{z}+\frac{1}{4} \operatorname{Re} \delta_{k 1} W_{e} q_{k}^{*} \\
& =(-1)^{k-1} J^{z}+\frac{1}{4} \operatorname{Re} \delta_{k 1} W_{e}\left[q_{b}^{*}+(-1)^{k-1} q_{c}^{*}\right]
\end{aligned}
$$

in the stationary state. From (2.28), $\mathbf{J}_{H k}$, which is included on the right-hand side of (4.16), reduces to the barotropic enstrophy flux,

$$
\mathbf{J}_{b}=\operatorname{Re}\left(\frac{\left|\psi_{b, x}\right|^{2}-\left|\psi_{b, y}\right|^{2}}{2}, \quad \psi_{b, x} \psi_{b, y}^{*}\right),
$$

as $F \rightarrow \infty$ for both the upper and lower layers $(k=1,2)$. From (2.29) and (4.4), the right-hand side of (4.16) for $k=1,2$ also yields the same form as $F \rightarrow \infty$, and (4.16) becomes

$$
\nabla \cdot \mathbf{J}_{b}=\operatorname{Re} W_{e} q_{b}^{*} .
$$

Equation (4.17) merely corresponds to the enstrophy equation of the barotropic mode. It can be shown that this is also the case for the harmonic wave. Thus, there is no inconsistency in the formulation in this section.

\section{b. Southward PV flux}

In this subsection, we examine the phase differences between $q_{b}$ and $q_{\beta}$ and between $W$ and $W_{\beta}$, based on numerical experiments. We drop the subscript $b$, which denotes the barotropic mode, in the rest of this section because the baroclinic mode does not appear explicitly as $F \rightarrow \infty$. As a typical example of $q$, the black contours in the left panels of Fig. 6 show $\operatorname{Re} q$ and $\operatorname{Im} q$ for $a=1$. Here $\operatorname{Im}$ denotes the imaginary part, and $\operatorname{Re} q$ and Im $q$ represent the snapshot of the lowest-order PV, $q^{(0)}=\operatorname{Re} q e^{-i t}$, at $t=0$ and $\pi / 2$, respectively. For comparison, the black contours in the right panels of Fig. 6 show the snapshot of $q_{\beta}^{(0)}=\operatorname{Re} q_{\beta} e^{-i t}$, at $t=0$ and $\pi / 2$. The region $y<0$ is not shown because $q$ and $q_{\beta}$ are symmetric about $y=0$. Because $q-q_{\beta}=q_{w}$ is imaginary from (4.8), $q^{(0)}$ is identical to $q_{\beta}^{(0)}$ at $t=0$ (Figs. 6a, b). Although $q^{(0)}$ increases from $t=0$ to $\pi / 2$ near $(x, y)=$ $(0,0), q_{\beta}^{(0)}$ becomes weakly negative at $t=\pi / 2$ in the same region (Figs. 6c,d). As the forcing attains a maximum at $t=0, q$ and $q_{\beta}$ are delayed and slightly advanced relative to the forcing. Because $q_{\beta}$ becomes negative when the planetary vorticity is advected northward [see the second equation in (4.8)], the change in $q_{\beta}$ from $t=0$ to $\pi / 2$ simply indicates that the northward flow is driven by the positive forcing.

As shown in (4.13), $W$ and $W_{\beta}$ are determined from the advection of $q$ and $q_{\beta}$ by $\psi$, respectively. When flow due to $\psi$ is in the downgradient direction of $q, W$ is positive, and vice versa. The blue contours in Fig. 6 indicate snapshots of $\psi^{(0)}=\psi e^{-i t}$. At $t=0$, the northward or northwestward flow near $(x, y)=(0,0)$ is oriented in the downgradient direction of both $q$ and $q_{\beta}$ (Figs. 6a,b). At $t=\pi / 2$, although the flow near $(x, y)=(0,0)$ continues to be oriented in the downgradient direction of $q$, it is oriented in the upgradient direction of $q_{\beta}$.

Figure 7 compares the snapshot of the nonlinear forcing,

$$
\begin{aligned}
& \Omega=-J\left(\psi^{(0)}, q^{(0)}\right)=W e^{-2 i t}-\frac{1}{2} J\left(\psi, q^{*}\right) \text { and } \\
& \Omega_{\beta}=-J\left(\psi^{(0)}, q_{\beta}^{(0)}\right)=W_{\beta} e^{-2 i t}-\frac{1}{2} J\left(\psi, q_{\beta}^{*}\right),
\end{aligned}
$$

for $a=1$ at $t=0$ (top panels), $\pi / 4$ (upper-middle panels), $\pi / 2$ (lower-middle panels), and $3 \pi / 4$ (bottom panels). Note that $\Omega$ and $\Omega_{\beta}$ include the forcing to the mean flow as well as $W$ and $W_{\beta}$. At both $t=0$ and $\pi / 2, \Omega$ is positive near $x=0$ (Figs. 7a,e), as expected from Figs. 6a and 6c. Similarly, $\Omega_{\beta}$ is positive at $t=0$ and negative at $t=\pi / 2$ near $x=0$ (Figs. 7b,f), as expected from Figs. $6 \mathrm{~b}$ and $6 \mathrm{~d}$. Because the frequency of the harmonic wave is twice as large as that of the primary wave, the interval between $t=0$ and $\pi / 2$ corresponds to half of the harmonic wave's period. Therefore, $W$ and $W_{\beta}$ are nearly out of phase with each other. Figure 8 compares the real and imaginary parts of $W$ and $W_{\beta}$ for $a=1$. We can confirm that $W$ and $W_{\beta}$ are nearly out of phase with each other, except in the region $0<x<1,1<y<2$. Figure 9 shows the horizontal distribution of the PV flux in the lower layer obtained from (4.14). The southward PV flux dominates, corresponding to the phase difference between $W$ and $W_{\beta}$. As $q^{(0)}$ includes a component proportional to $W_{e}$ (see (4.7) and (4.8)), the discontinuities in $x$ and $y$ derivatives of $W_{e}$ given by (3.5) cause discontinuities in $\Omega$ and $W$ along $x=0$ and $y=0$ (Figs. $7 \mathrm{c}, \mathrm{e}$ ). However, because $\psi_{b}^{h}$ and $\psi_{c}^{h}$ are expressed in terms of the forcing by the primary wave using (3.3) and (3.4), $\psi_{b}^{h}$ and $\psi_{c}^{h}$ are continuous in $x$ and $y$, causing no serious problems to other variables.

\section{c. Semianalytic solution}

In this subsection, we confirm that the results obtained in the previous subsection are consistent with the basic 
(a)

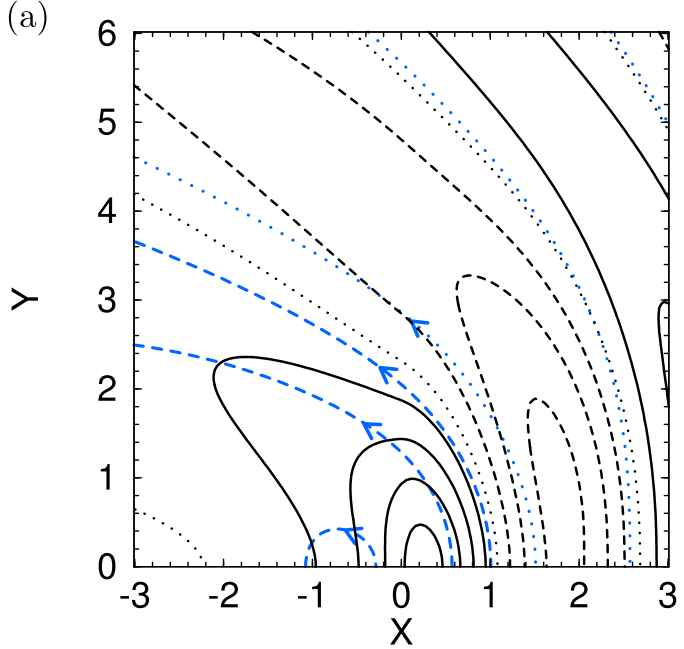

(c)

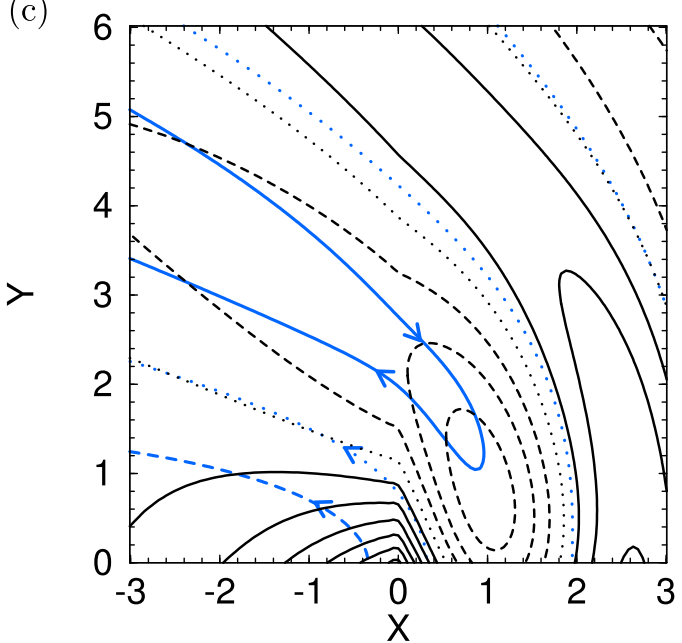

(b)

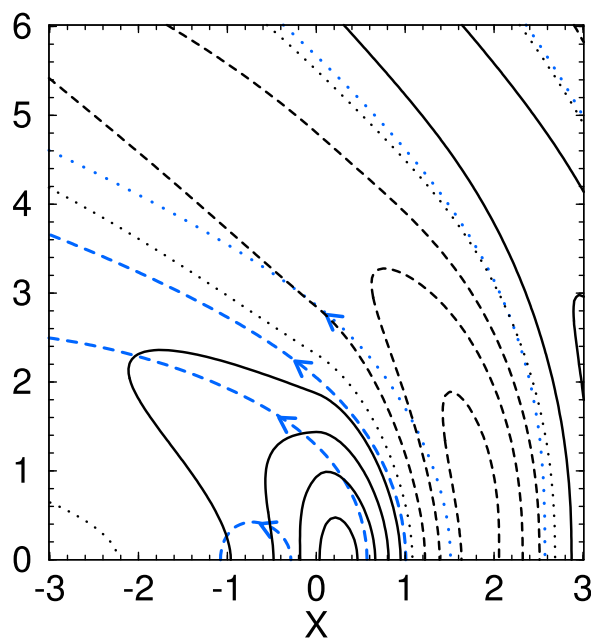

(d)

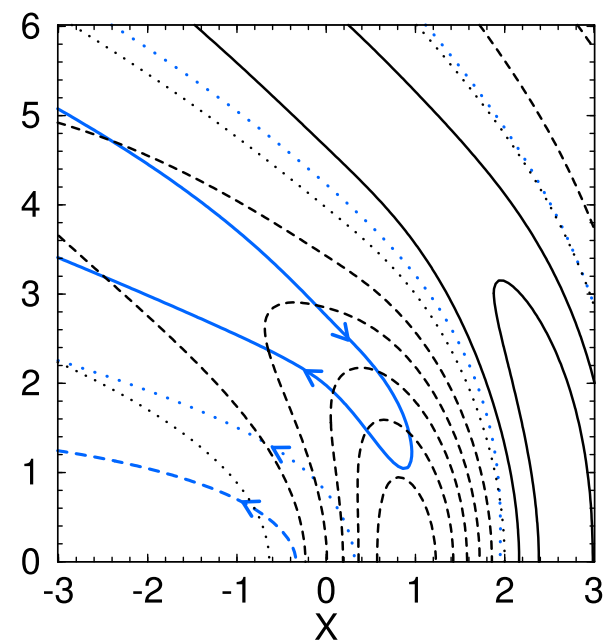

FIG. 6. Comparison of (left) $q^{(0)}$ and (right) $q_{\beta}^{(0)}$. The black contours indicate snapshots of $q^{(0)}$ and $q_{\beta}^{(0)}$ at $t=$ (top) 0 and (bottom) $\pi / 2$. Snapshots of $\psi^{(0)}$ are superimposed by blue contours. Blue arrows indicate the direction of the flow. Contour intervals are 1.5 and 1.8 for the black and blue contours, respectively.

features of Rossby waves. We also examine the effects of the resonant triad interaction (Longuet-Higgins and Gill 1967) on the PV flux. Substituting (3.5) into (2.12) and taking the Fourier transform in $x$, we obtain

$$
\tilde{\psi}_{y y}-\lambda^{2} \tilde{\psi}=\frac{2 i a A}{a^{2} k^{2}+1} e^{-|y| / a},
$$

where

$$
\lambda=\sqrt{k(k+2)}
$$

and the tilde denotes the Fourier component,

$$
\tilde{\psi}=\int_{-\infty}^{\infty} \psi e^{-i k x} d x
$$

Then we obtain

$$
\begin{aligned}
\tilde{\psi} & =\sum_{m=1}^{2} R_{m} e^{i l_{m}|y|}, \\
\tilde{q} & =-\sum_{m=1}^{2}\left(k^{2}+l_{m}^{2}\right) R_{m} e^{i l_{m}|y|}, \text { and } \\
\tilde{q}_{\beta} & =\sum_{m=1}^{2} 2 k R_{m} e^{i l_{m}|y|},
\end{aligned}
$$

where

$$
l_{1}=i \lambda, \quad l_{2}=\frac{i}{a},
$$

$R_{1}(k)=\frac{2 i a^{2} A}{\lambda\left(a^{2} \lambda^{2}-1\right)\left(a^{2} k^{2}+1\right)}, \quad$ and 

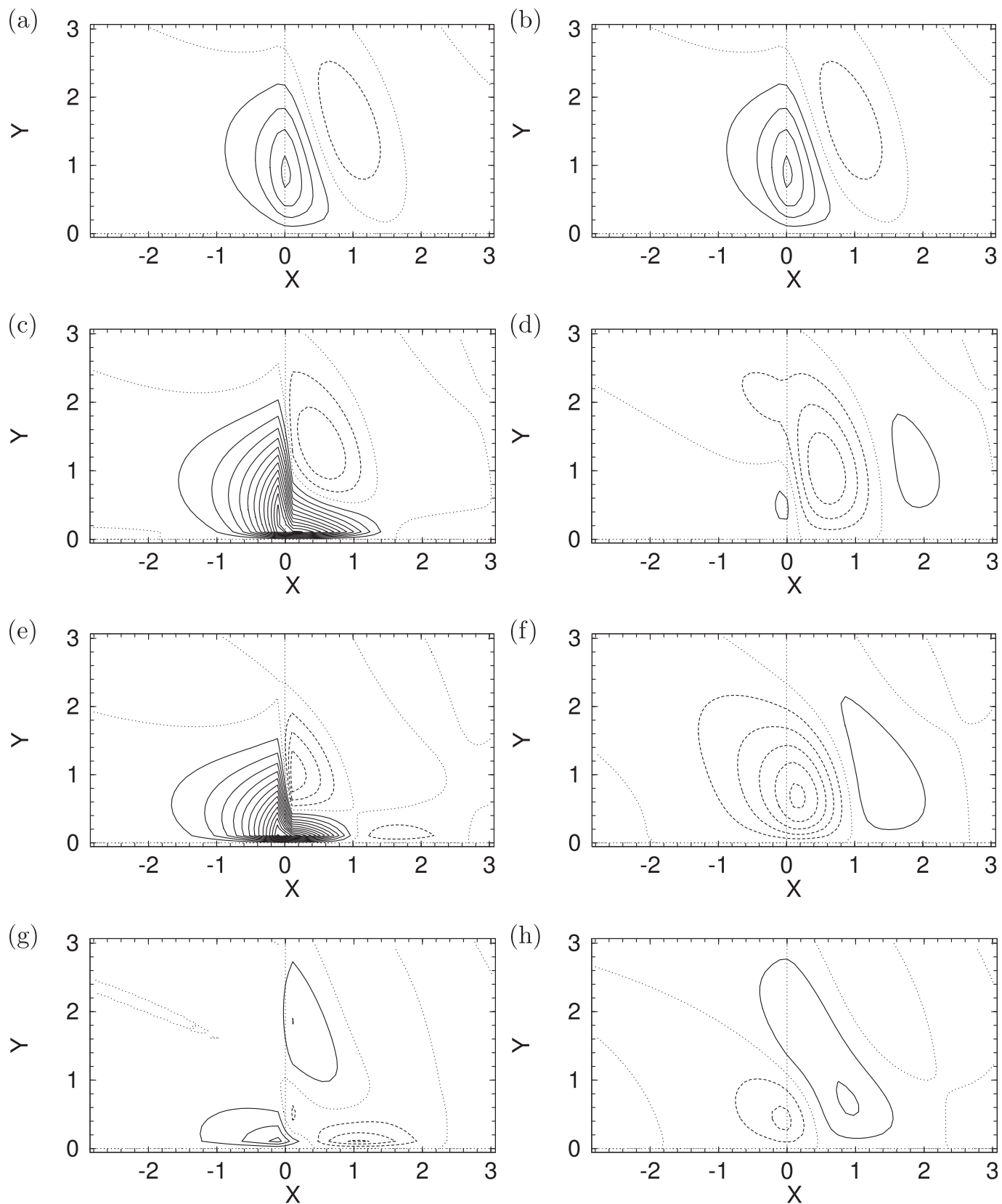

FIG. 7. Snapshots of (left) $\Omega$ and (right) $\Omega_{\beta}$ at $t=$ (first row) 0 , (second row) $\pi / 4$, (third row) $\pi / 2$, and (fourth row) $3 \pi / 4$. Contour interval is 2 .

$$
R_{2}(k)=-\frac{2 i a^{3} A}{\left(a^{2} \lambda^{2}-1\right)\left(a^{2} k^{2}+1\right)} .
$$

As $\left(k, l_{1}\right)$ is real and satisfies the dispersion relation in the interval $-2<k<0$, we can decompose $\tilde{q}$ and $\tilde{q}_{\beta}$ into the resonant (or free) and forced components:

$$
\begin{aligned}
\tilde{q} & =\tilde{q}_{R}+\tilde{q}_{F}, \quad \text { and } \\
\tilde{q}_{\beta} & =\tilde{q}_{R}+\tilde{q}_{\beta F},
\end{aligned}
$$

where the subscripts $R$ and $F$ denote the resonant and forced components, respectively, and

$$
\begin{gathered}
\tilde{q}_{R}=-\sum_{m=1}^{2} s_{m}\left(k^{2}+l_{m}^{2}\right) R_{m} e^{i l_{m}|y|}, \\
\tilde{q}_{F}=-\sum_{m=1}^{2}\left(1-s_{m}\right)\left(k^{2}+l_{m}^{2}\right) R_{m} e^{i l_{m}|y|}, \\
\tilde{q}_{\beta F}=\sum_{m=1}^{2} 2\left(1-s_{m}\right) k R_{m} e^{i l_{m}|y|}, \quad \text { and }
\end{gathered}
$$



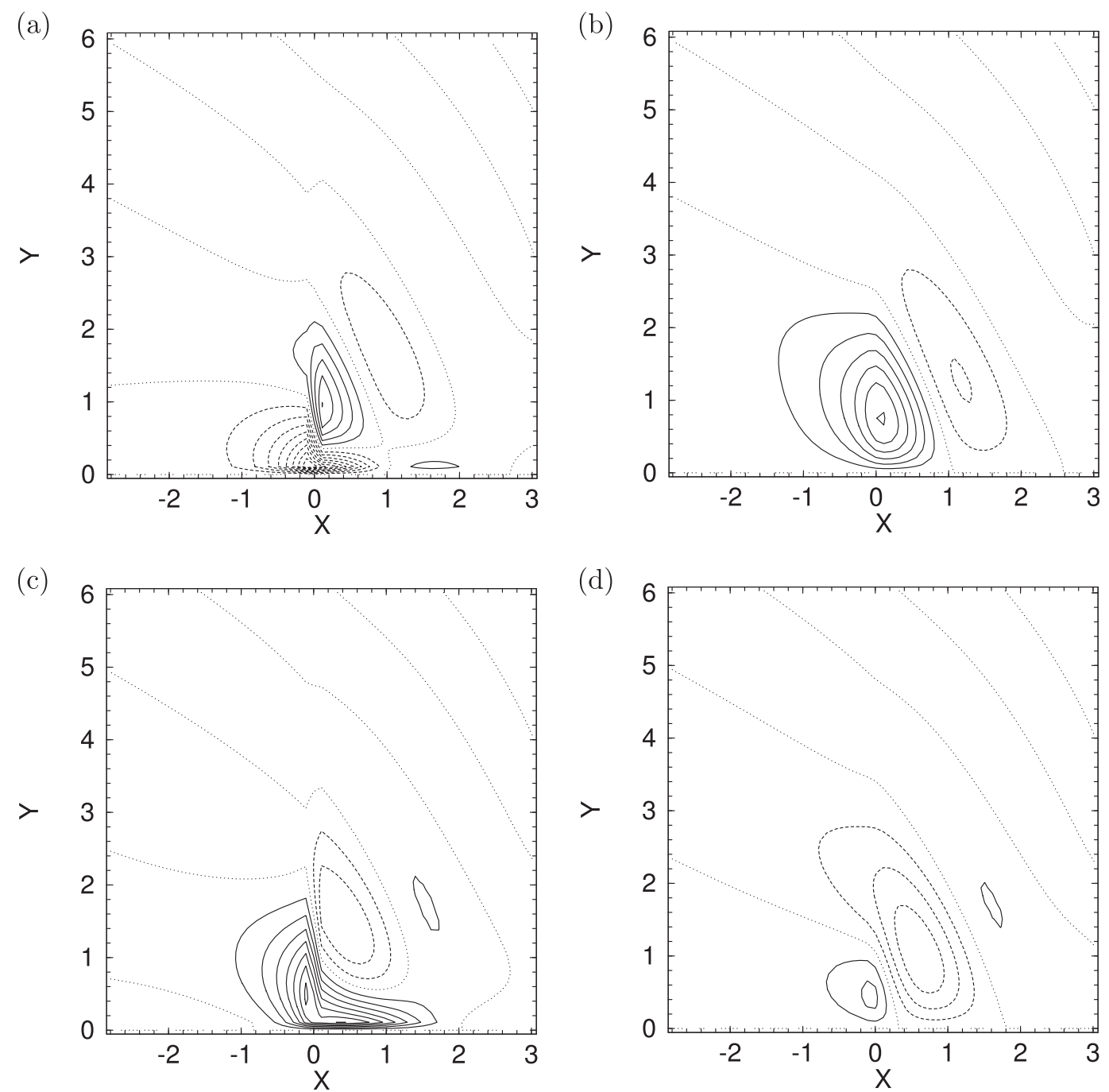

FIG. 8. Contours of (a) $\operatorname{Re} W$, (b) $\operatorname{Re} W_{\beta}$, (c) $\operatorname{Im} W$, and (d) $\operatorname{Im} W_{\beta}$. Contour interval is 1.5.

$s_{m}(k)=\left\{\begin{array}{ll}1 & \text { when } m=1 \text { and }-2 \leq k \leq 0 \\ 0 & \text { otherwise }\end{array}\right.$.

The resonant component of $\tilde{q}_{\beta}$ is the same as that of $\tilde{q}$, because $k^{2}+l_{1}^{2}+2 k=0$.

Using (4.13) and the identity,

$$
\tilde{f} g=\frac{1}{2 \pi} \int_{-\infty}^{\infty} \tilde{f}\left(k-k^{\prime}\right) \tilde{g}\left(k^{\prime}\right) d k^{\prime},
$$

we obtain

$$
\begin{aligned}
\tilde{W}= & \frac{1}{4 \pi} \int_{k / 2}^{\infty} \sum_{m=1}^{2} \sum_{n=1}^{2}\left(k^{\prime} l_{n}^{\prime \prime}-k^{\prime \prime} l_{m}^{\prime}\right) \\
& \times\left(k^{2}+l_{m}^{\prime 2}-k^{\prime \prime 2}-l_{n}^{\prime \prime 2}\right) R_{m}^{\prime} R_{n}^{\prime \prime} e^{i\left(l_{m}^{\prime}+l_{n}^{\prime \prime}\right) y} d k^{\prime} \text { and }
\end{aligned}
$$

$$
\begin{aligned}
\tilde{W}_{\beta}= & -\frac{1}{2 \pi} \int_{k / 2}^{\infty} \sum_{m=1}^{2} \sum_{n=1}^{2}\left(k^{\prime} l_{n}^{\prime \prime}-k^{\prime \prime} l_{m}^{\prime}\right) \\
& \times\left(k^{\prime}-k^{\prime \prime}\right) R_{m}^{\prime} R_{n}^{\prime \prime} e^{i\left(l_{m}^{\prime}+l_{n}^{\prime \prime}\right) y} d k^{\prime},
\end{aligned}
$$

in $y>0$, where

$$
\begin{aligned}
k^{\prime \prime} & =k-k^{\prime}, \\
l_{m}^{\prime} & =l_{m}\left(k^{\prime}\right), \quad l_{n}^{\prime \prime}=l_{n}\left(k^{\prime \prime}\right), \quad \text { and } \\
R_{m}^{\prime} & =R_{m}\left(k^{\prime}\right), \quad R_{n}^{\prime \prime}=R_{n}\left(k^{\prime \prime}\right) .
\end{aligned}
$$

Because $\tilde{W}$ and $\tilde{W}_{\beta}$ are antisymmetric about $y=0$, the form in $y<0$ is not shown. As we did for the PV, we decompose $W$ and $W_{\beta}$ into resonant and forced components:

$$
\begin{aligned}
W & =-J\left(\psi, q_{R}\right)-J\left(\psi, q_{F}\right) \\
& =W_{R}+W_{F}, \quad \text { and }
\end{aligned}
$$




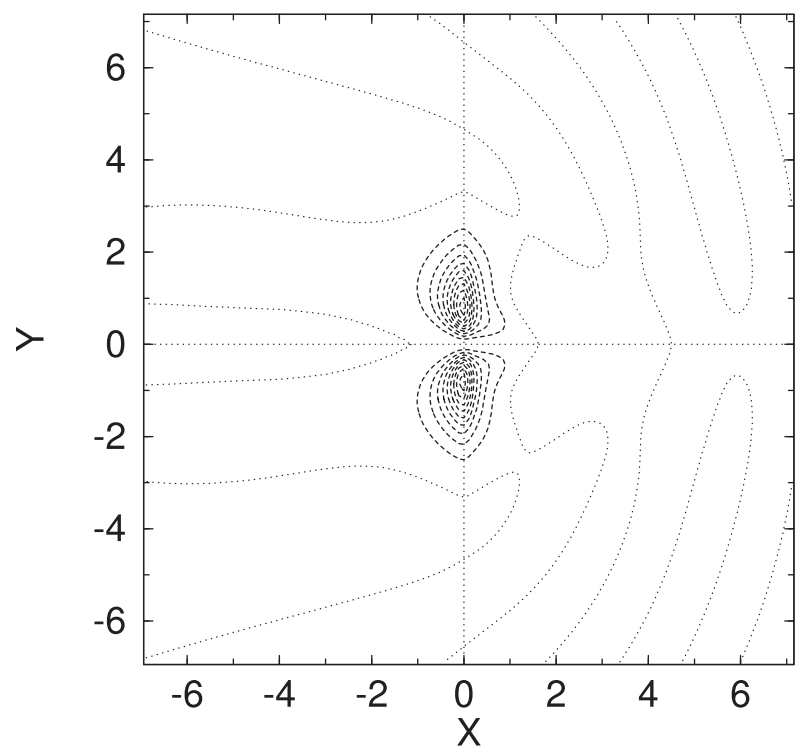

FIG. 9. Contours of the meridional PV flux in the lower layer in the limit as $F \rightarrow \infty$. Contour interval is 0.15 .

$$
\begin{aligned}
W_{\beta} & =-J\left(\psi, q_{R}\right)-J\left(\psi, q_{\beta F}\right) \\
& =W_{R}+W_{\beta F} .
\end{aligned}
$$

Substituting (4.33) and (4.34) into (4.14) and (4.15), we can also decompose $Y_{2}^{h 0}$ and $Y_{b}^{h 0}$ into resonant and forced components:

$$
\begin{aligned}
Y_{2}^{h 0} & =-\frac{1}{2} \operatorname{Re} \eta^{h 0} W_{R}^{*}-\frac{1}{2} \operatorname{Re} \eta^{h 0} W_{\beta F}^{*} \\
& =Y_{R}^{h 0}+Y_{\beta F}^{h 0}, \quad \text { and } \\
Y^{h 0} & =-\frac{1}{2} \operatorname{Re} \eta^{h 0} W_{R}^{*}-\frac{1}{2} \operatorname{Re} \eta^{h 0} W_{F}^{*} \\
& =Y_{R}^{h 0}+Y_{F}^{h 0} .
\end{aligned}
$$

Note that the subscript $b$ in $Y_{b}^{h 0}$ is dropped here.

Equation (4.31) implies that two waves with wavenumbers of $\mathbf{k}_{m}^{\prime}=\left(k^{\prime}, l_{m}^{\prime}\right)$ and $\mathbf{k}_{n}^{\prime \prime}=\left(k^{\prime \prime}, l_{n}^{\prime \prime}\right)$ drive motions with the wavenumber of $\mathbf{k}_{m}^{\prime}+\mathbf{k}_{n}^{\prime \prime}=\left(k, l_{m}^{\prime}+l_{n}^{\prime \prime}\right)$. The form on the right-hand side of (4.31) is reminiscent of the triad interaction of Rossby waves (Longuet-Higgins and Gill 1967). [Note that $W$ is antisymmetric in $y$. Then it can be shown that motions increase linearly in $y$ if resonance occurs.] However, $l_{1}^{\prime}$ and $l_{1}^{\prime \prime}$ can take only negative values ( $l_{2}^{\prime}$ and $l_{2}^{\prime \prime}$ are imaginary) in the region $y>0$, as the group velocity should be positive there [see (4.22)]. Then the resonance condition is not satisfied because $\mathbf{k}_{1}^{\prime}+\mathbf{k}_{1}^{\prime \prime}$ is located outside the circle in Fig. 10, which represents the dispersion curve of the primary wave (vector $\mathbf{O C}$ in Fig. 10; see the caption of Fig. 10 for proof), while the dispersion curve of the harmonic wave is located inside this circle (small circle in Fig. 10). Thus,

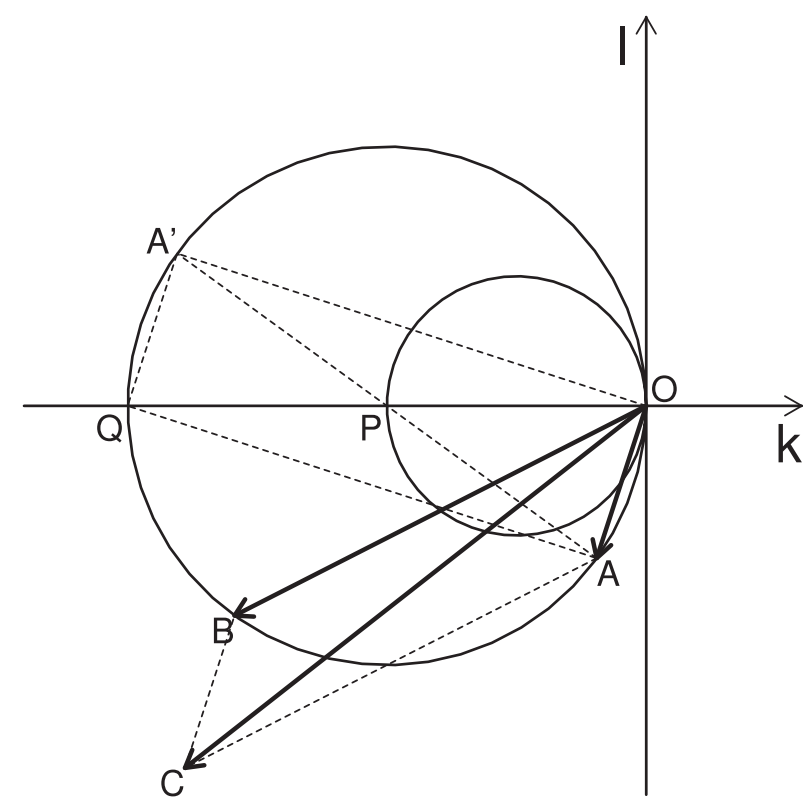

FIG. 10. Schematic of a triad of barotropic Rossby waves. The large and small circles indicate the dispersion curves of the primary and harmonic waves, respectively. Point $\mathrm{O}$ is the origin, $\mathbf{O A}=\mathbf{k}_{1}^{\prime}$ and $\mathbf{O B}=\mathbf{k}_{1}^{\prime \prime}$ are the wavenumbers of the primary wave, and $\mathbf{O C}=\mathbf{k}_{1}^{\prime}+\mathbf{k}_{1}^{\prime \prime}$. Point $\mathrm{P}$ is the center of the large circle, and $\mathrm{A}^{\prime}$ is the crossing of the line AP and the large circle. When B is located at $\mathrm{A}^{\prime}$, $\mathrm{C}$ is located at $\mathrm{Q}$, which is the crossing of the line $\mathrm{OP}$ and the large circle. When $\mathrm{B}$ is located on the $\operatorname{arc} \mathrm{OA} \mathrm{A}^{\prime}, \mathrm{C}$ is located outside the large circle. Thus, if $\mathrm{A}$ and $\mathrm{B}$ are located in the region $l<0, \mathrm{C}$ is located outside the large circle.

we cannot focus on a specific triad but instead must consider all triads including both resonant and forced components.

Figure 11a shows the distribution of the real and imaginary parts of $\tilde{q}_{F}, \tilde{q}_{\beta F}$, and $\tilde{q}_{R}$ at $y=0$. Because $\operatorname{Re} \tilde{q}_{F}=\operatorname{Re} \tilde{q}_{\beta F}=\operatorname{Im} \tilde{q}_{R}=0$, they are not shown. The results shown in Fig. 11a can be interpreted by analogy with an oscillator forced by a periodic forcing, as the equation of the PV can be expressed in the form of the forced oscillator:

$$
\frac{d \hat{q}^{(0)}}{d t}=-i \omega_{0} \hat{q}^{(0)}+\hat{W}_{e} e^{-i t}
$$

where the hat denotes the two-dimensional Fourier component,

$$
\hat{q}^{(0)}=\iint_{-\infty}^{\infty} q^{(0)} e^{-i(k x+l y)} d x d y,
$$

and

$$
\omega_{0}=-\frac{2 k}{k^{2}+l^{2}}
$$


corresponds to the natural frequency of the oscillator. The resonance condition, $\omega_{0}=1$, is satisfied on the dispersion curve (the circle in Fig. 12). Because of the character of the forced oscillator, $\hat{q}^{(0)}$ is in phase with $\hat{W}_{e}$ when $\omega_{0}=1$, whereas the phase of $\hat{q}^{(0)}$ relative to $\hat{W}_{e}$ is $\pi / 2$ for $\omega_{0}<1$ (regions B and C in Fig. 12) and $-\pi / 2$ for $\omega_{0}>1$ (region A). Hence, $\tilde{q}_{R}$, which corresponds to resonant $\hat{q}^{(0)}$, is in phase with $\tilde{W}_{e}$ (dotted line in Fig. 11). The phase of $\tilde{q}_{F}$, which is the sum of $\hat{q}^{(0)}$ at $\omega_{0} \neq 1$, is mostly $\pi / 2$ except for the interval $-2<k<-1$ (solid line in Fig. 11). From (2.18), (4.7), and (4.38), we obtain

$$
\hat{q}_{\beta}^{(0)}=\omega_{0} \hat{q}^{(0)} .
$$

Hence $\tilde{q}_{\beta F}$ is imaginary and out of phase with $\tilde{q}_{F}$ when $k>0$, owing to the contribution of $\hat{q}^{(0)}$ in the region C where $\omega_{0}<0$ (dashed line in Fig. 11).

Figure 13 shows the horizontal distribution of $q_{F}, q_{\beta F}$, and $q_{R}$ for $a=1$, which are obtained numerically using the inverse Fourier transform of (4.27)-(4.29). [Note that singularities of $O\left(k^{-1 / 2}\right)$ at $k=0,-2$ in (4.23) vanish when the inverse Fourier integral is performed with a complex variable $\theta=\cos ^{-1}(k+1)$.] As expected from Fig. 11a, the phase relative to the forcing at $(x, y)=(0,0)$ is $\pi / 2$ for $q_{F}$ (Figs. 13a,d) and 0 for $q_{R}$ (Figs. 13c,f). Similarly, Fig. 11b shows the distribution of $\operatorname{Im} \tilde{\psi}_{F}$ and $\operatorname{Re} \tilde{\psi}_{R}$. Note that $\operatorname{Re} \tilde{\psi}_{F}=\operatorname{Im} \tilde{\psi}_{R}=0$. Because of the difference by the factor $-\left(k^{2}+l_{m}^{2}\right)$ between $\psi$ and $q, \tilde{\psi}_{F}$ and $\tilde{\psi}_{R}$ are mostly out of phase with $\tilde{q}_{F}$ and $\tilde{q}_{R}$, respectively. Also, $\tilde{\psi}_{R}$ has a larger amplitude for long waves compared to $\tilde{q}_{R}$ (dotted line in Fig. 11b).
Figure 14 shows the horizontal distribution of $\psi_{R}$ and $\psi_{F}$ for $a=1$. Because the wavenumber of the long wave is more meridional than the short wave (see vectors OA and $\mathbf{O B}$ in Fig. 10), contours of $\psi_{R}$ are more zonal compared to $q_{R}$ (see gray lines in Figs. 13c and 14a). The phase relative to the forcing at $(x, y)=(0,0)$ is $\pi$ for $\psi_{R}$ and $-\pi / 2$ for $\psi_{F}$. The distributions of $q, q_{\beta}$, and $\psi$ in Fig. 6 are consistent with those in Figs. 13 and 14.

Figures $15 \mathrm{a}$ and $15 \mathrm{~b}$ compare the zonal integrals of each term in (4.35) and (4.36), respectively. For the barotropic component of the PV flux, $Y_{F}^{h 0}$ is positive and contributes to the northward PV flux (dashed line in Fig. 15b). Although the phase difference between $\tilde{q}_{R}$ and $\tilde{q}_{F}$ is $\pi / 2$ except for the factor $e^{i l_{m}|y|}, Y_{R}^{h 0}$ is southward (dotted line in Fig. 15b). The integral of $Y^{h 0}$ over the entire model domain is 0.079 and positive, as we considered in section $2 \mathrm{~b}$. The reason why $Y_{R}^{h 0}$ has the opposite sign to $Y_{F}^{h 0}$ may be qualitatively explained as follows. As we have seen, the distribution of $q_{F}, q_{R}$, and $\psi$ may be expressed in an idealized form:

$$
\begin{aligned}
q_{F} & =i B e^{-(\kappa|x|+\lambda y)}, \\
q_{R} & =C e^{-i\left(k_{1} x+l_{1} y\right)}, \quad \text { and } \\
\psi & =-D e^{-i\left(k_{2} x+l_{2} y\right)},
\end{aligned}
$$

where all parameters are real and positive. We assume for simplicity that $k_{1}, l_{1}, k_{2}$, and $l_{2}$ vary slowly in space and $C$ and $D$ decrease slowly from $(x, y)=(0,0)$, although these assumptions are not exactly valid. Substituting (4.39)-(4.41) into (4.33) yields

$$
W_{F}=\left\{\begin{array}{lc}
-\frac{1}{2}\left(k_{2} \lambda-l_{2} \kappa\right) B D e^{-(\kappa x+\lambda y)-i\left(k_{2} x+l_{2} y\right)} & \text { in } \quad x>0 \\
-\frac{1}{2}\left(k_{2} \lambda+l_{2} \kappa\right) B D e^{\kappa x-\lambda y-i\left(k_{2} x+l_{2} y\right)} & \text { in } \quad x<0
\end{array},\right. \text { and }
$$

$W_{R}=\frac{1}{2}\left(k_{1} l_{2}-l_{1} k_{2}\right) C D e^{-i\left[\left(k_{1}+k_{2}\right) x+\left(l_{1}+l_{2}\right) y\right]}$.

Because $\left(k_{1}, l_{1}\right)$ (i.e., the wavenumber of $\left.q_{R}\right)$ is more zonal than $\left(k_{2}, l_{2}\right)$ (i.e., the wavenumber of $\psi$ ) the term in brackets of (4.43) is positive. In the region $x>0, l_{2}$ tends to be smaller than $k_{2}$ (see blue contours in Fig. 13), mostly due to the contribution from short waves and partly due to $\psi_{F}$. Hence, the term in brackets in (4.42) is mostly positive. Then $W_{R}$ is out of phase with $W_{F}$ near $(x, y)=$ $(0,0)$, producing $Y_{R}^{h 0}$, which has the opposite sign to $Y_{F}^{h 0}$.

In contrast, the PV flux $Y_{2}^{h 0}$ in the lower layer becomes southward because of $Y_{R}^{h 0}$ (dotted and solid lines in
Fig. 15a). The forced component $Y_{\beta F}^{h 0}$ also contributes to the southward PV flux near $y=0$, where $q_{\beta F}$ is out of phase with $q_{F}$ (dashed line in Fig. 15a). Thus, the southward PV flux in the lower layer is produced by the difference between $q_{F}, q_{R}$, and $q_{\beta F}$, which can be interpreted only by the basic features of the Rossby waves. Qualitatively the same results can be obtained for the other values of $a$.

\section{Effects of stratification}

To examine the effects of stratification on the PV flux, we consider the second-lowest terms of the perturbation 
(a)

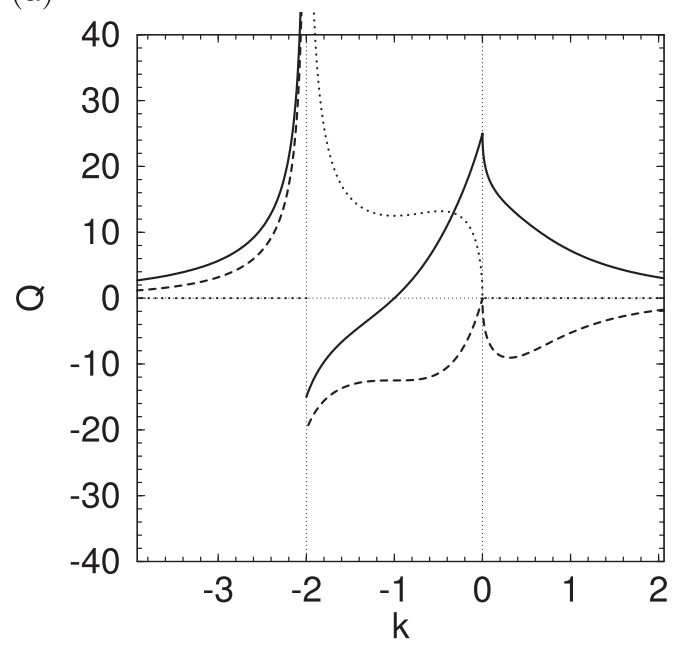

(b)

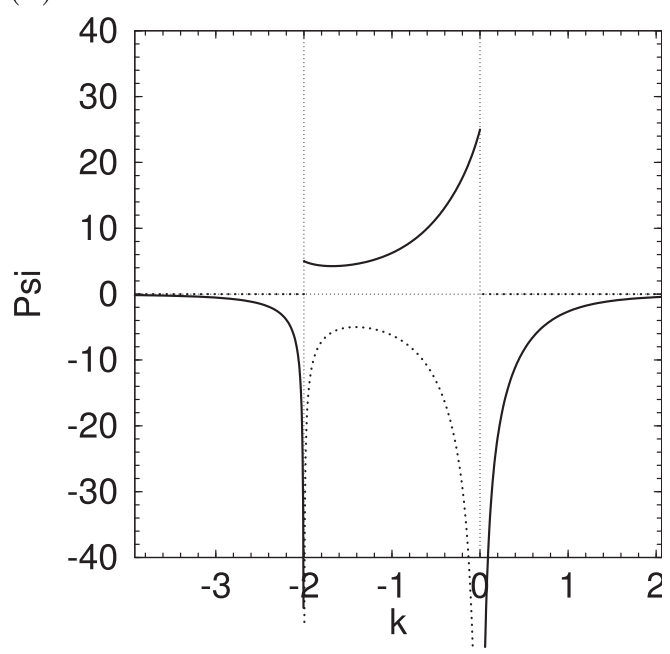

FIG. 11. Plots of (a) $\operatorname{Im} \tilde{q}_{F}$ (solid), $\operatorname{Im} \tilde{q}_{\beta F}$ (dashed), and $\operatorname{Re} \tilde{q}_{R}$ (dotted), and (b) $\operatorname{Im} \tilde{\psi}_{F}$ (solid) and $\operatorname{Re} \tilde{\psi}_{R}$ (dotted) at $y=0$ as a function of $k$.

expansions in (4.1) and (4.2). Substituting (4.3)-(4.6) into (2.16) and (2.17), and taking $O\left(F^{-1}\right)$ terms, we obtain

$$
\begin{aligned}
& w_{b}^{h^{\prime}}=-J\left(\psi_{c}^{\prime}, q_{w}\right) \text { and } \\
& w_{c}^{h^{\prime}}=-J\left(\psi_{b}^{0}, q_{c}^{\prime}\right)-J\left(\psi_{c}^{\prime}, q_{\beta}\right)-J\left(\psi_{c}^{\prime}, q_{w}\right) .
\end{aligned}
$$

Because (4.4) and (4.8) indicate that both $\psi_{c}^{\prime}$ and $q_{w}$ are proportional to $W_{e}$, the last terms on the right-hand side of (5.1) and (5.2) vanish, implying that

$$
w_{b}^{h^{\prime}}=\eta_{b}^{h^{\prime}}=0 .
$$

By expanding $\eta_{k}^{M}$ in (2.31) into vertical modes, we can decompose the lower-layer PV flux into the contributions from $\eta_{b}^{h}$ and $\eta_{c}^{h}$ :

$$
\begin{aligned}
Y_{2}^{h} & =-\frac{1}{2} \operatorname{Re} \eta_{b}^{h} w_{2}^{h^{*}}+\frac{1}{2} \operatorname{Re} \eta_{c}^{h} w_{2}^{h^{*}} \\
& =Y_{2 b}^{h}+Y_{2 c}^{h} .
\end{aligned}
$$

Combining (5.1)-(5.4), and keeping terms of $O\left(F^{-1}\right)$ or lower, yields

$$
\begin{aligned}
Y_{2 b}^{h}= & -\frac{1}{2} \operatorname{Re} \eta_{b}^{h 0} W_{\beta}^{*}-\frac{1}{F} \operatorname{Re} \eta_{b}^{h 0}\left[J\left(\psi_{b}^{0}, W_{e, x}\right)\right. \\
& \left.-J\left(\psi_{b, x}^{0}, W_{e}\right)\right] \text { and } \\
Y_{2 c}^{h}= & \frac{1}{2 F} \operatorname{Re} \eta_{c}^{h^{\prime}} W_{\beta}^{*} .
\end{aligned}
$$

We have used the relation $q_{c}^{\prime}=-2 i \psi_{c, x}^{\prime}$, which can be derived from (2.13) and (2.19). The first and second terms on the right-hand side of (5.5) represent the PV flux of $O(1)$ discussed in previous sections and the correction of
$O\left(F^{-1}\right)$ to $w_{2}^{h}$, respectively. Although the baroclinic wave is evanescent, (5.6) indicates that the interaction between $\eta_{c}^{h}$ and the barotropic forcing produces the baroclinic PV flux. Taking the $O(1)$ terms in (2.15) and using (2.32), (4.11), and (4.12), we can express $\eta_{c}^{h^{\prime}}$ as

$$
\eta_{c}^{h^{\prime}}=\frac{W_{x}-W_{\beta, x}}{4} .
$$

The solid, dashed, and dotted lines in Fig. 16 show the area integrals of $Y_{2}^{h}, Y_{2 b}^{h}$, and $Y_{2 c}^{h}$, which are directly calculated from the numerical solution as a function of $F^{1 / 2}$,

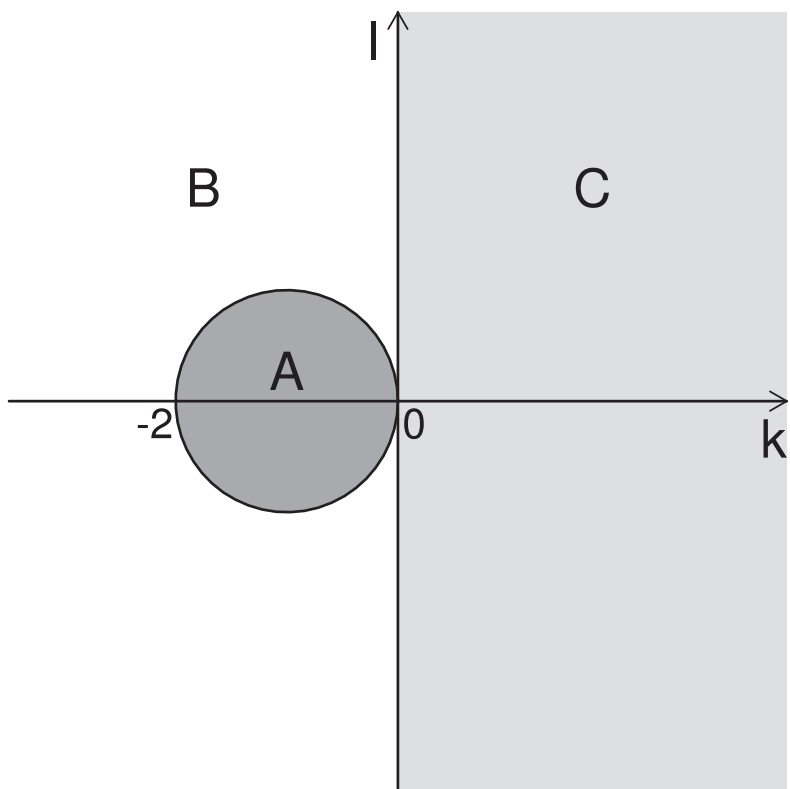

FIG. 12. Definitions of regions A, B, and C. The solid circle indicates the dispersion curve of the primary wave. 
(a)

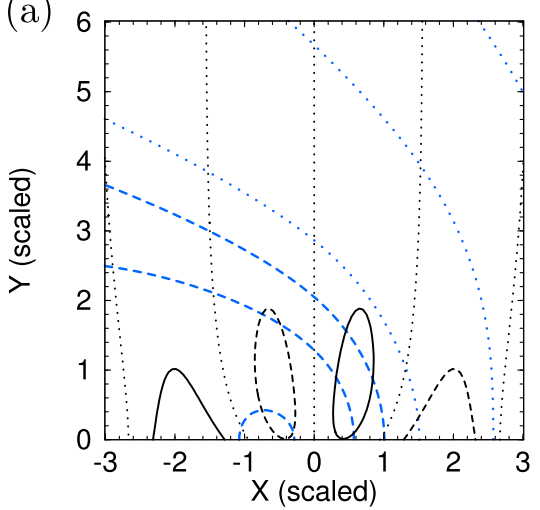

(d)

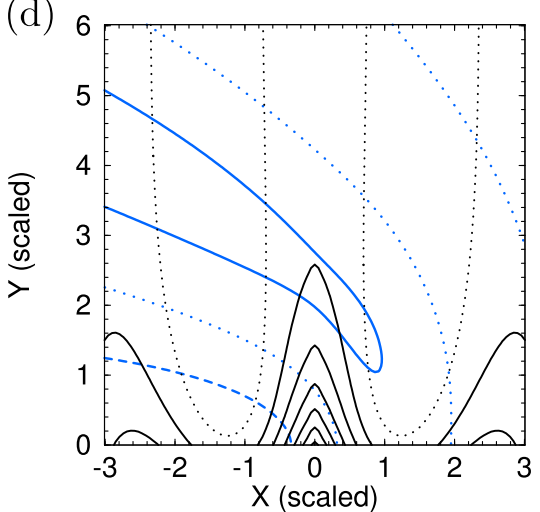

(b)

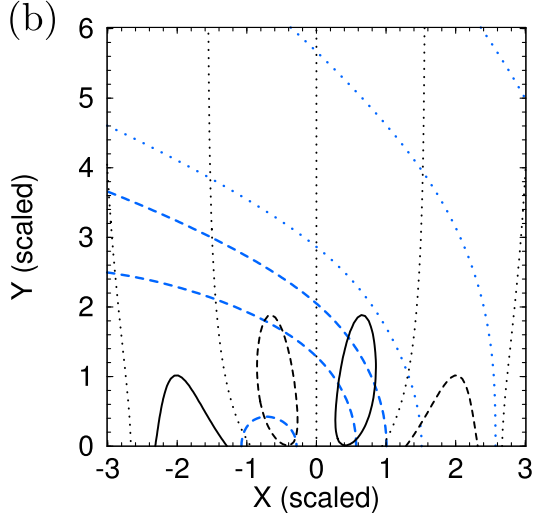

(e)

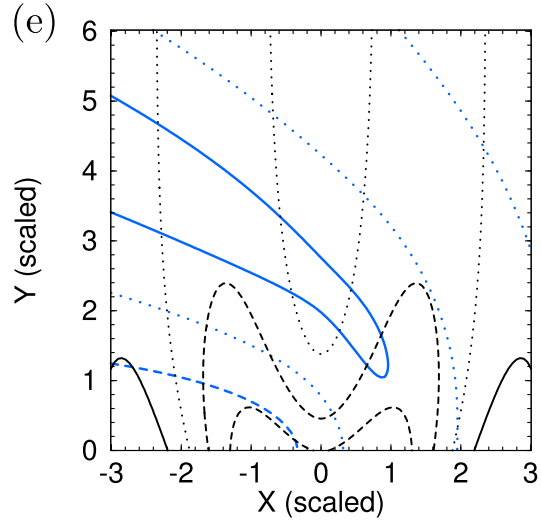

(c)

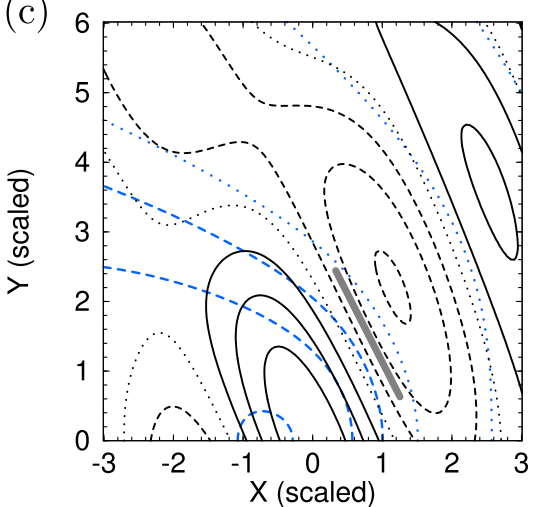

(f)

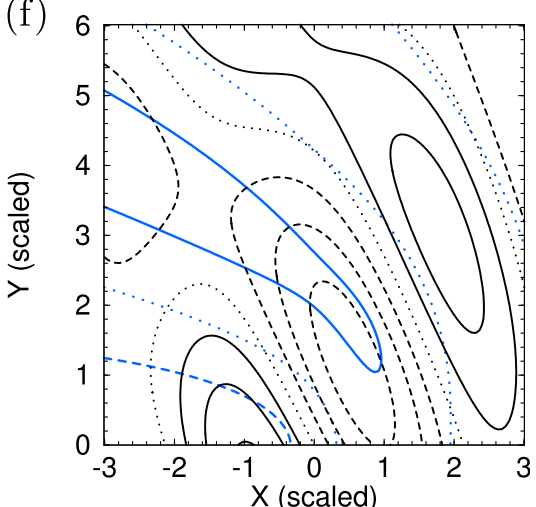

FIG. 13. Comparison of (left) $q_{F} e^{-i t}$, (center) $q_{\beta F} e^{-i t}$, and (right) $q_{R} e^{-i t}$ at $t=$ (top) 0 and (bottom) $\pi / 2$ shown by black contours. Snapshots of $\psi^{(0)}$ are superimposed by blue contours. Contour intervals are 1.5 and 1.8 for the black and blue contours, respectively. In (c) the gray solid line indicates the typical orientation of the contours of $q_{R} e^{-i t}$.

respectively. For comparison, $Y_{2 b}^{h}$ and $Y_{2 c}^{h}$ estimated from (5.5) and (5.6) are also shown by the thick dashed and thick dotted lines, respectively. The PV flux estimated from (5.5) and (5.6) qualitatively agrees with that obtained from the numerical solution except for the interval near $F=1$. By comparing (5.6) and the first term of (5.5), we see that $Y_{2 c}^{h}$ represents the effects of the change in lower-layer motions by stratification. The southward PV flux in the lower layer is weakened by $Y_{2 c}^{h}$, which is northward, for both $a=0.5$ and 1 (dotted lines in Fig. 16). This corresponds to the weakening of the lower-layer motions by stratification. The second term on the right-hand side of (5.5) weakens the southward PV flux, which is produced by the first term of (5.5), for $a=0.5$ and strengthens it for $a=1$ (dashed lines in Fig. 16). The change in $Y_{2 b}^{h}$ with $F^{1 / 2}$ dominates over that in $Y_{2 c}^{h}$ for $a>1.5$ (not shown), corresponding to the increase of the southward PV flux with decreasing $F^{1 / 2}$ shown in Fig. 4b.

\section{Summary}

To better understand the wave-mean interaction in the western boundary region, we examined the effects of stratification and nonlinearity on the PV flux produced by the forced Rossby waves. The perturbation analysis shows that the primary wave is excited directly by the forcing, whereas the harmonic wave is excited by the nonlinear interaction of the primary wave. The harmonic wave produces the southward PV flux in the lower layer, whereas the primary wave produces the northward PV flux in the upper layer. Because of the conventional nonacceleration theorem, no meridional PV flux is produced by the primary wave in the lower layer, in which the external forcing vanishes.

The southward PV flux dominates in the lower layer in the wide parameter range typical for the western boundary region. This may be counterintuitive, as the volume integral of the PV flux should be northward according to the enstrophy equation. Even in the limit of infinitesimally weak stratification, the amplitude of the PV flux remains on the same order as that obtained for finite stratification. The horizontal scale of the PV flux is nearly proportional to $a$, except for the case where $F$ is close to unity.

Equations of the perturbation PV are greatly simplified in the limit of the weak stratification. In this limit, 

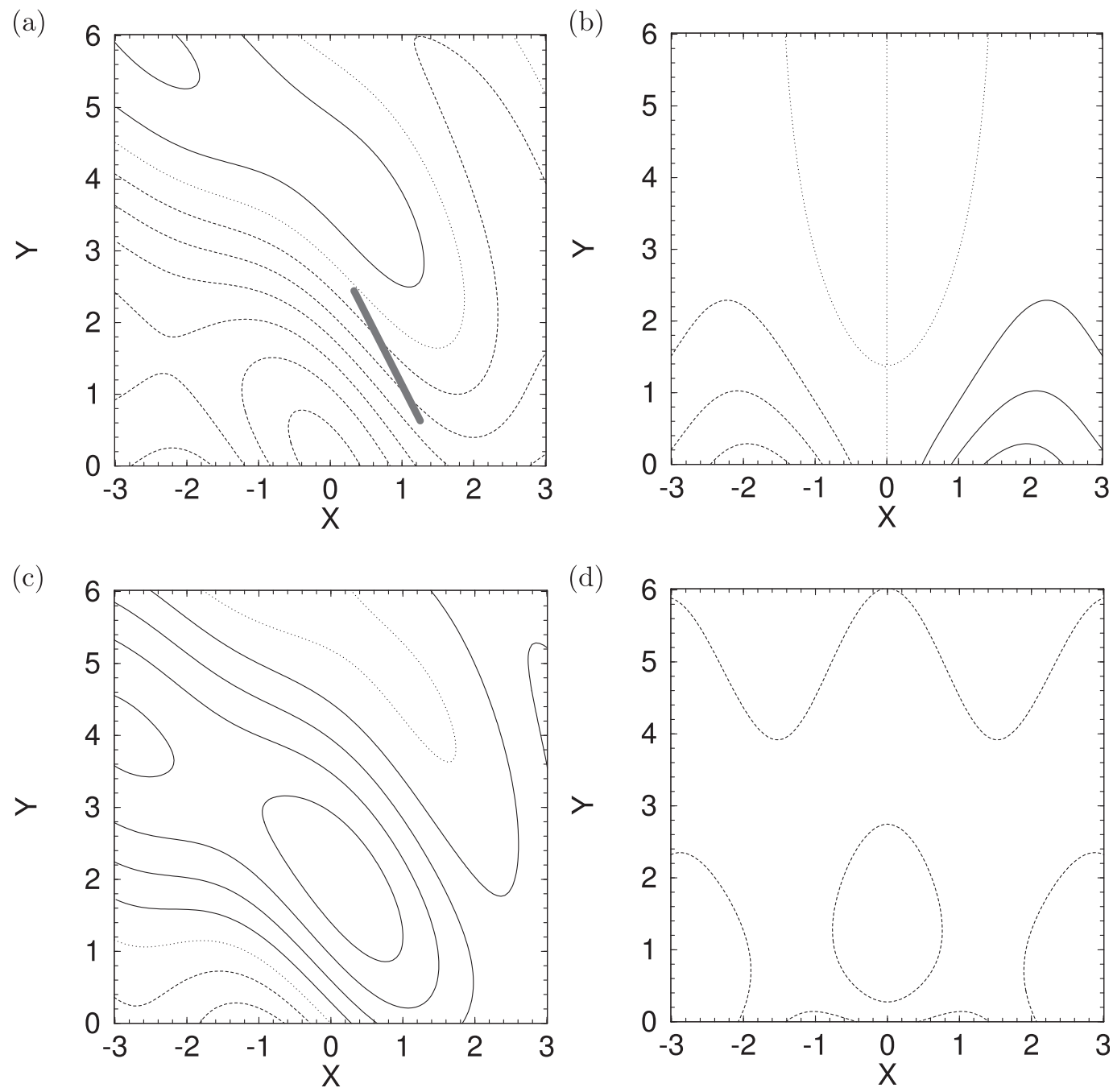

FIG. 14. Snapshots of (left) $\psi_{R} e^{-i t}$ and (right) $\psi_{F} e^{-i t}$ at $t=$ (top) 0 and (bottom) $\pi / 2$. Contour interval is 0.8 . In (a) the gray solid line indicates the typical orientation of the contours of $q_{R} e^{-i t}$ in Fig. 13c.

stratification has almost no effect on the flow, except that it isolates the lower layer from the direct effects of the external forcing. That is, the PV in the lower layer is determined only by the advection of the planetary vorticity. It is shown that a resonant triad interaction of Rossby waves is absent. Various triads of resonant (or free) and forced waves are substantial contributors to the PV flux. The southward PV flux is produced because the nonlinear forcing to the lower layer tends to be out of phase with the barotropic component of the nonlinear forcing. Although the phase of the nonlinear forcing is not determined by a simple process, it is explained only by basic features of Rossby waves and does not depend on details of experimental conditions. The difference between the forced and resonant components of the PV is a key feature that determines the phase of the nonlinear forcing (Figs. 6 and 13). The difference of dominant wavenumbers between $\psi$ and $q$ shown in Fig. 13 is also important. One might expect that the southward PV flux may be reproduced by the interaction between two or three waves that are periodic in $x$, making a simpler interpretation of the southward PV flux possible. However, such combinations of the waves could not be found. This is probably due to the fact that the above-noted features of $\psi$ and $q$ are essentially caused by the superposition of infinite number of resonant and forced waves.

The effects of finite stratification on the PV flux are qualitatively consistent with the perturbation analysis with respect to $F^{-1}$, except for the case where $F$ is close to unity. Because $\psi_{c}$ increases logarithmically as $F \rightarrow 1$ (i.e., $\omega \rightarrow \omega_{c}$ ), $\psi_{k}$ cannot be approximated by the asymptotic series of $F^{-1}$ like (4.1) as $F \rightarrow 1$. Except for this case, stratification weakens the response of lower-layer 
(a)

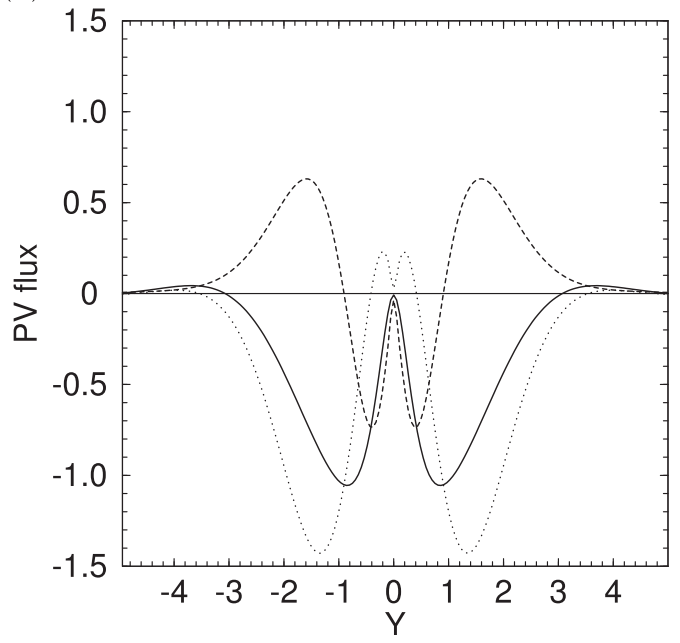

(b)

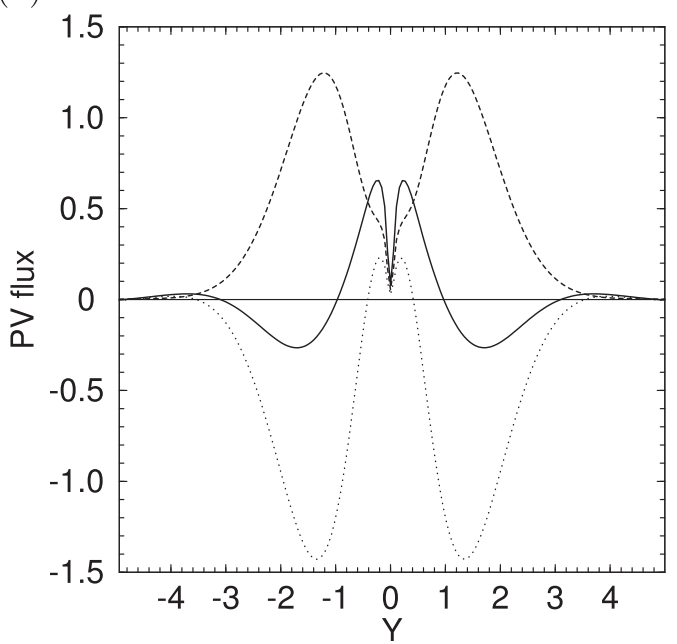

FIG. 15. Meridional distributions of the zonally integrated meridional PV flux. (a) Solid, dotted, and dashed lines indicate $Y_{2}^{h 0}, Y_{R}^{h 0}$, and $Y_{\beta F}^{h 0}$, respectively. (b) The solid, dotted, and dashed lines indicate $Y^{h 0}, Y_{R}^{h 0}$, and $Y_{F}^{h 0}$, respectively.

motions to the nonlinear forcing, whereas it weakens or strengthens the nonlinear forcing, depending on the value of $a$. When $a$ is smaller or larger than about 1.5 , the net effect of stratification is to weaken or strengthen the PV flux in the lower layer, respectively.

The direction of the PV flux obtained in the present study is consistent with that obtained in an eddyresolving model by Holland and Rhines (1980) in both the upper and lower layers. Results of the present study may contribute to the understanding of the driving mechanism of the recirculation gyres. In the present study, the external forcing was of single sign. When the forcing changes sign in space, the meridional PV flux also changes sign in space as expected from the enstrophy constraint in section $2 \mathrm{~b}$. However, the basic features of the PV flux remain unchanged. It has not been confirmed that the mechanism of the southward PV flux proposed in the present study is substantial in the real ocean or in eddy-resolving numerical models. This is left for future research. (a)

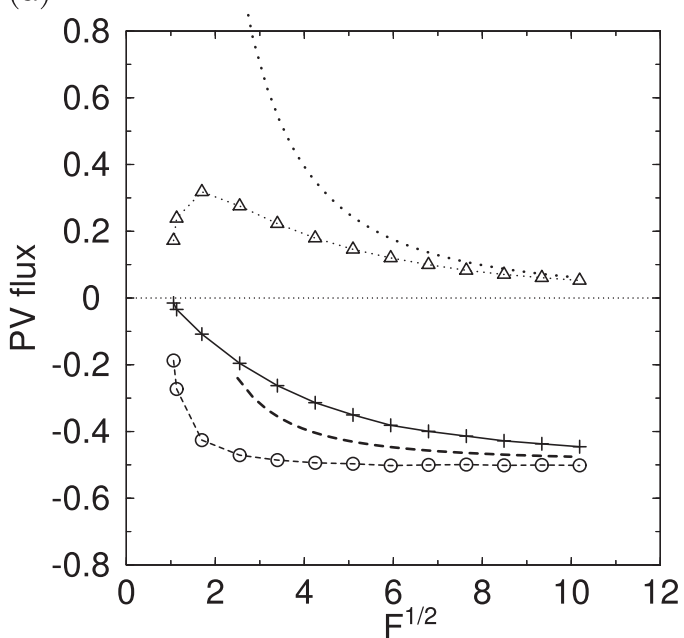

(b)

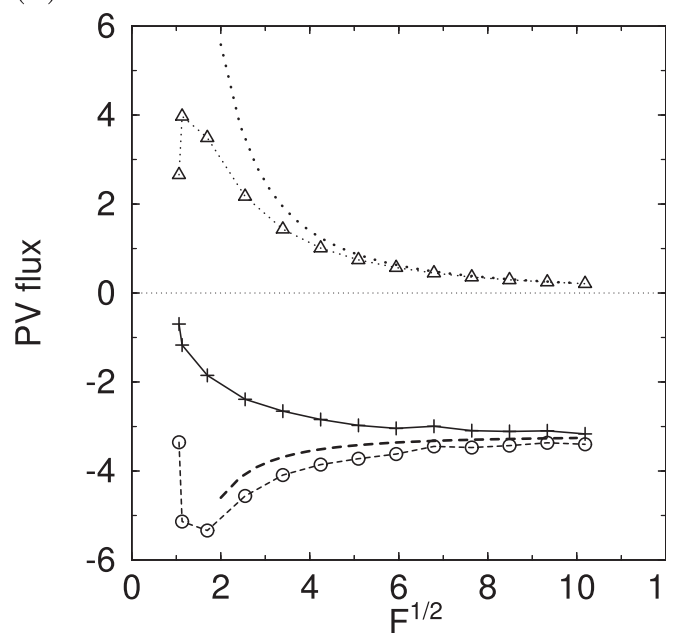

FIG. 16. Plots of the meridional PV flux, which is integrated in the entire domain, as a function of $F^{1 / 2}$ for $a=$ (a) 0.5 and (b) 1 . Solid lines with crosses, dashed lines with circles, and dotted lines with triangles indicate $Y_{2}^{h}, Y_{2 b}^{h}$, and $Y_{2 c}^{h}$, respectively. The thick dashed and dotted lines indicate $Y_{2 b}^{h}$ and $Y_{2 c}^{h}$ obtained by the perturbation analysis, respectively. 
Acknowledgments. The present study was supported by JSPS KAKENHI Grants JP23654167 and JP17K05649 and the Collaborative Research Program of the Research Institute for Applied Mechanics, Kyushu University.

\section{REFERENCES}

Bower, A. S., and N. G. Hogg, 1992: Evidence for barotropic wave radiation from the Gulf Stream. J. Phys. Oceanogr., 22, 42-61, https:// doi.org/10.1175/1520-0485(1992)022<0042:EFBWRF>2.0.CO;2.

Haidvogel, D. B., and P. B. Rhines, 1983: Waves and circulation driven by oscillatory winds in an idealized ocean basin. Geophys. Astrophys. Fluid Dyn., 25, 1-63, https://doi.org/10.1080/ 03091928308221747.

- , H. G. Arango, K. Hedstrom, A. Beckmann, P. MalanotteRizzoli, and A. F. Shchepetkin, 2000: Model evaluation experiments in the North Atlantic basin: Simulations in nonlinear terrain-following coordinates. Dyn. Atmos. Oceans, 32, 239-281, https://doi.org/10.1016/S0377-0265(00)00049-X.

Hogg, N. G., 1981: Topographic waves along $70^{\circ} \mathrm{W}$ on the continental rise. J. Mar. Res., 39, 627-649.

- 1983: A note on the deep circulation of the western North Atlantic: Its nature and causes. Deep-Sea Res., 30, 945-961, https://doi.org/10.1016/0198-0149(83)90050-X.

__, 1988: Stochastic wave radiation by the Gulf Stream. J. Phys. Oceanogr., 18, 1687-1701, https://doi.org/10.1175/ 1520-0485(1988)018<1687:SWRBTG > 2.0.CO;2.

— 1992: On the transport of the Gulf Stream between Cape Hatteras and the Grand Banks. Deep-Sea Res., 39, 1231-1246, https://doi.org/10.1016/0198-0149(92)90066-3.

— , R. S. Pickart, R. Hendry, and W. Smethie, 1986: On the northern recirculation gyre of the Gulf Stream. Deep-Sea Res., 33, 1139-1166, https://doi.org/10.1016/0198-0149(86)90017-8.

Holland, W. R., and P. B. Rhines, 1980: An example of eddy-induced ocean circulation. J. Phys. Oceanogr., 10,1010-1031, https://doi. org/10.1175/1520-0485(1980)010<1010:AEOEIO > 2.0.CO;2.

Imawaki, S., 1985: Features of mesoscale eddies in the deep mid-ocean of the western North Pacific. Deep-Sea Res., 32, 599-611, https://doi.org/10.1016/0198-0149(85)90046-9.

Jayne, S. R., and Coauthors, 2009: The Kuroshio Extension and its recirculation gyres. Deep-Sea Res., 56, 2088-2099, https://doi. org/10.1016/j.dsr.2009.08.006.

Kawai, H., 1972: Hydrography of the Kuroshio Extension. Kuroshio, H. Stommel and K. Yoshida, Eds., University of Tokyo Press, 235-354.
Longuet-Higgins, M., and A. Gill, 1967: Resonant interactions between planetary waves. Proc. Roy. Soc. London, 299A, 120 144, https://doi.org/10.1098/rspa.1967.0126.

Malanotte-Rizzoli, P., N. G. Hogg, and R. E. Young, 1995: Stochastic radiation by the Gulf Stream: Numerical experiments. Deep-Sea Res., 42, 389-423, https://doi.org/10.1016/0967-0637 (95)00001-M.

Mizuta, G., 2009: Rossby wave radiation from an eastward jet and its recirculations. J. Mar. Res., 67, 185-212, https://doi.org/ 10.1357/002224009789051227.

_ 2014: The potential-vorticity flux by the barotropic Rossby wave excited by the oscillatory forcing-Basic features. Rep. Res. Inst. Appl. Mech. Kyushu Univ., 146, 75-85.

_ 2018: Upgradient and downgradient potential vorticity fluxes produced by forced Rossby waves. Part I: Basic experiments. J. Phys. Oceanogr., 48, 1191-1209, https://doi.org/10.1175/ JPO-D-17-0197.1.

Plumb, R. A., 1986: Three-dimensional propagation of transient quasi-geostrophic eddies and its relationship with the eddy forcing of the time-mean flow. J. Atmos. Sci., 43, 1657-1678, https://doi.org/10.1175/1520-0469(1986) 043<1657:TDPOTQ > 2.0.CO;2.

Rhines, P. B., and W. R. Holland, 1979: A theoretical discussion of eddy-driven mean flows. Dyn. Atmos. Oceans, 3, 289-325, https://doi.org/10.1016/0377-0265(79)90015-0.

— and W. R. Young, 1982: A theory of wind-driven circulation. I. Mid-ocean gyres. J. Mar. Res., 40, 559-596.

Richardson, P. L., 1983: A vertical section of eddy kinetic energy though the Gulf Stream system. J. Geophys. Res., 88, 27052709, https://doi.org/10.1029/JC088iC04p02705.

Thompson, R., 1977: Observations of Rossby waves near site D. Prog. Oceanogr., 7, 135-162, https://doi.org/10.1016/0079-6611 (77)90003-9.

_ , and J. R. Luyten, 1976: Evidence of bottom-trapped topographic Rossby waves from single moorings. Deep-Sea Res. Oceanogr. Abstr., 23, 629-635, https://doi.org/10.1016/0011-7471(76)90005-X.

Waterman, S., and S. R. Jayne, 2011: Eddy-mean flow interactions in the along-stream development of a western boundary current jet: An idealized model study. J. Phys. Oceanogr., 41, 682-707, https://doi.org/10.1175/2010JPO4477.1.

_- and —_, 2012: Eddy-driven recirculations from a localized transient forcing. J. Phys. Oceanogr., 42, 430-447, https://doi.org/10.1175/JPO-D-11-060.1.

Worthington, L. V., 1976: On the North Atlantic Circulation. The Johns Hopkins Oceanographic Studies, Vol. 6, The Johns Hopkins University Press, 110 pp. 